\title{
Redefined "Maclaurin Symmetric Mean Aggregation Operators Based on Cubic Pythagorean Linguistic Fuzzy Numbers"
}

\author{
Muhammad Naeem $\mathbb{D}^{1},{ }^{1}$ Shahzaib Ashraf $\mathbb{D}^{2},{ }^{2}$ Saleem Abdullah $\mathbb{D}^{3},{ }^{3}$ and F. M. AL-Harbi ${ }^{4}$ \\ ${ }^{1}$ Deanship of Combined First Year, Umm Al-Qura University, Makkah, Saudi Arabia \\ ${ }^{2}$ Department of Mathematics and Statistics, Bacha Khan University, Charsadda 24420, Khyber Pakhtunkhwa, Pakistan \\ ${ }^{3}$ Department of Mathematics, Abdul Wali Khan University, Mardan 23200, Khyber Pakhtunkhwa, Pakistan \\ ${ }^{4}$ Deanship of Combined First Year, Umm Al-Qura University, Makkah, Saudi Arabia \\ Correspondence should be addressed to Shahzaib Ashraf; shahzaibashraf@bkuc.edu.pk
}

Received 13 January 2021; Revised 26 March 2021; Accepted 15 April 2021; Published 17 May 2021

Academic Editor: Mingwei Lin

Copyright (C 2021 Muhammad Naeem et al. This is an open access article distributed under the Creative Commons Attribution License, which permits unrestricted use, distribution, and reproduction in any medium, provided the original work is properly cited.

\begin{abstract}
In this study, we highlight the errors in Sections 2.3, 2.4, 3, and 4 in the article by Fahmi et al. (J Ambient Intell Human Comput (2020). https://doi.org/10.1007/s12652-020-02272-9) by counter definitions and theorems. We find that the definition of cubic Pythagorean fuzzy set (CPFS) (Definition 2.3.1) and operational laws (Definition 2.3.2) violates the rules to consider the membership and nonmembership functions, and then, we redefined the corrected definition and their operations for CPFS. Furthermore, we redefine the concept of cubic Pythagorean linguistic fuzzy set (CPLFS) and their basic operational laws. In addition, we find that Sections 3 and 4 (consist of a list of Maclaurin symmetric mean (MSM) and dual MSM aggregation operators) are invalid, and then, we redefined the list of updated MSM and dual MSM aggregation operators in correct way. Finally, we established the numerical application of the proposed improved algorithm using cubic Pythagorean linguistic fuzzy information to show the applicability and effectiveness of the proposed technique.
\end{abstract}

\section{Introduction}

Due to the increasing complexity of the system, it is difficult for the decision maker to select the best alternative/object from a set of attractive options in the real world. However, it is hard to summarize, but it is not incredible to achieve the best single objective. A large number of multicriteria decision-making problems exist in decision-making, where the criteria are found to be uncertain, ambiguous, imprecise, and vague. As a result, the crisp set appears to be ineffective in dealing with this uncertainty and imprecision in the data and can be easily dealt with by using fuzzy information. To deal with such uncertainty and ambiguity, Zadeh [1] presented the mathematical notion of fuzzy set (FS) which has been defined by using the membership function of the element. Various researchers have discovered the utility of the fuzzy set in a variety of fields, including decision-making, medical diagnosis, engineering, socioeconomic, and finance problems.
With the continuous process of human practice, decision-making problems have become more and more complicated, and many extended forms of fuzzy sets [1] have been proposed, such as the bipolar soft sets [2], the intuitionistic fuzzy (IF) sets [3], the interval-valued intuitionistic fuzzy sets [4], Pythagorean fuzzy (PyF) sets [5], picture fuzzy $(\mathrm{PF})$ sets $[6,7]$, and spherical fuzzy (SF) sets [8-10]. Many decision-making techniques under Pythagorean fuzzy information are established, for example, Wang et al. [11, 12] presented the novel decision-making techniques under Pythagorean fuzzy interactive Hamacher power and interaction power Bonferroni mean aggregation operators are proposed and discussed their applications in multiple attribute decision-making problems. Khan et al. [13] presented the decision-making method based on probabilistic hesitant fuzzy rough information. In [14], Ashraf et al. worked on sine trigonometric aggregation operator for Pythagorean fuzzy numbers; in [15], Batool et al. developed new models 
for decision making under Pythagorean hesitant fuzzy numbers. Khan et al. used the Dombi t-norms and t-conorms to Pythagorean fuzzy numbers and defined Pythagorean fuzzy Dombi aggregation operators [16].

The cubic Pythagorean fuzzy set (CPFS) is a well reputed structure of fuzzy sets, proposed by Abbas et al. [17] in 2019 to tackle the uncertainty in decision-making problems. Talukdar and Dutta [18] presented the distance measures under CPFS information. Fahmi et al. [19] proposed the decision-making technique using cubic Pythagorean linguistic fuzzy sets. The main objective of this note is to highlight the error in Sections 2.3, 2.4, 3, and 4 in the study by Fahmi et al. [19] by counterdefinitions and countertheorems.

\section{Preliminaries}

We initiate with rudimentary concept of fuzzy set, cubic set, intuitionistic fuzzy set, Pythagorean fuzzy set, and cubic Pythagorean fuzzy set that are required for the rest of this paper.

Definition 1 (see [1]). A fuzzy set (FS) $\mathscr{F}$ in arbitrary set $U \neq \phi$ has the form

$$
\mathscr{F}=\{\langle v, \mu(v)\rangle \mid v \in U\},
$$

where $\mu(v) \in[0,1]$ is represented by the positive membership grade.

Definition 2 (see [20]). An interval-valued fuzzy set (IVFS) $\mathscr{F}$ in a universe set $U$ is an object having the form

$$
\mathscr{F}=\left\{\left\langle v,\left[\mu^{-}(v), \mu^{+}(v)\right]\right\rangle \mid v \in U\right\},
$$

where $\mu(v) \in[0,1]$ is represented by the positive membership grade.

Definition 3 (see [21]). A cubic set $\mathscr{F}$ in a universe set $U$ is an object having the form

$$
\mathscr{F}=\left\{\left\langle v,\left[\mu^{-}(v), \mu^{+}(v)\right], \mu(v)\right\rangle \mid v \in U\right\},
$$

where $\left[\mu^{-}(v), \mu^{+}(v)\right] \subseteq[0,1] \in \mathrm{IVFS}$ and $\mu(v) \in[0,1] \in$ FS.

Definition 4 (see [3]). An intuitionistic FS $\mathscr{F}_{s}$ in a universe set $U$ is an object having the form

$$
\mathscr{F}_{s}=\{\langle v, \mu(v), \partial(v)\rangle \mid v \in U\},
$$

where $\mu(v) \in[0,1]$ are positive and $\partial(v) \in[0,1]$ are negative membership grades, respectively. In addition, $0 \leq \mu(v)+\partial(v) \leq 1, \forall v \in U$.

Definition 5 (see [5]). A Pythagorean FS $\mathscr{F}_{s}$ in a universe set $U$ is an object having the form

$$
\mathscr{F}_{s}=\{\langle v, \mu(v), \partial(v)\rangle \mid v \in U\},
$$

where $\mu(v) \in[0,1]$ are positive and $\partial(v) \in[0,1]$ are negative membership grades, respectively. In addition, $0 \leq \mu^{2}(v)+\partial^{2}(v) \leq 1, \forall v \in U$.
Definition 6 (see [22]). An interval-valued Pythagorean FS (IVPFS) $\mathscr{F}_{s}$ in a universe set $U$ is an object having the form

$$
\mathscr{F}_{s}=\left\{\left\langle v,\left[\mu^{-}(v), \mu^{+}(v)\right],\left[\partial^{-}(v), \partial^{+}(v)\right]\right\rangle \mid v \in U\right\},
$$

where $\left[\mu^{-}(v), \mu^{+}(v)\right] \subseteq[0,1]$ are positive and $\left[\partial^{-}(v), \partial^{+}(v)\right] \subseteq[0,1]$ are negative membership grades, respectively. In addition, $0 \leq\left(\mu^{+}(v)\right)^{2}+\left(\partial^{+}(v)\right)^{2} \leq 1, \forall v \in U$.

Definition 7 (see [17]). A cubic Pythagorean fuzzy set $\mathscr{F}_{s}$ in a universe set $U$ is an object having the form

$$
\mathscr{F}=\left\{\left\langle v,\left(\left[\mu^{-}(v), \mu^{+}(v)\right],\left[\partial^{-}(v), \partial^{+}(v)\right]\right),(\mu(v), \partial(v))\right\rangle \mid v \in U\right\},
$$

where $\left[\mu^{-}(v), \mu^{+}(v)\right],\left[\partial^{-}(v), \partial^{+}(v)\right] \subseteq[0,1] \in$ IVPFS and $\mu(v), \partial(v) \in[0,1] \in$ PFS.

Maclaurin symmetric mean (MSM) is established by Maclaurin [23] and defined as follows.

Definition 8 (see [23]). Take any collection of nonnegative elements $h_{j}(j \in \mathbb{N})$ and $k=1,2, \ldots, n$. If

$$
\operatorname{MSM}^{(k)}\left(h_{1}, h_{2}, \ldots, h_{n}\right)=\left(\sum_{1 \leq i_{1} \leq i_{2} \leq \ldots \leq i_{k} \leq n} \frac{\prod_{j=1}^{k} h_{i_{j}}}{\mathbb{C}_{n}^{k}}\right)^{(1 / k)},
$$

then $\mathrm{MSM}^{(k)}$ is said to be MSM operator, where $\mathbb{C}_{n}^{k}$ is the binomial coefficient and $\left(i_{1}, i_{2}, \ldots, i_{k}\right)$ traversal all the k-tuple combination of $(1,2, \ldots, n)$.

Dual Maclaurin symmetric mean (DMSM) is established by Wei et al. [24] and defined as follows.

Definition 9 (see [24]). Take any collection of nonnegative elements $h_{j}(j \in \mathbb{N})$ and $k=1,2, \ldots, n$. If

$$
\operatorname{DMSM}^{(k)}\left(h_{1}, h_{2}, \ldots, h_{n}\right)=\frac{1}{k}\left(\prod_{1 \leq i_{1} \leq i_{2} \leq \cdots \leq i_{k} \leq n}\left(\sum_{j=1}^{k} h_{i_{j}}\right)^{\left(1 / C_{n}^{k}\right)}\right) \text {, }
$$

then $\operatorname{MDSM}^{(k)}$ is said to be DMSM operator, where $\mathbb{C}_{n}^{k}$ is the binomial coefficient and $\left(i_{1}, i_{2}, \ldots, i_{k}\right)$ traversal of all the k-tuple combination of $(1,2, \ldots, n)$.

\section{Counter Section 2.3 of [19]}

This section recalls the discussion of cubic Pythagorean fuzzy numbers (CPFN) and their basic operations proposed by Fahmi et al. [19].

Definition 2.3.1 in [19] proposed the definition of CPFS which is described as follows.

Definition 10 (see [19]). A CPFS $\mathscr{F}$ in arbitrary set $U \neq \phi$ has the form

$$
\mathscr{F}=\left\{\left\langle v,\left[\mu^{-}(4 v), \mu^{+}(v)\right], \mu(v)\right\rangle \mid v \in U\right\},
$$

where $\left[\mu^{-}(v), \mu^{+}(v)\right] \subseteq[0,1]$ and $\mu(v) \in[0,1]$ represent the positive and negative grades of memberships. 
For whole study, the list of cubic Pythagorean fuzzy sets are represented by $\operatorname{CPFS}(U)$. For simplicity, we write $\left\{\left[\mu^{-}(v), \mu^{+}(v)\right], \mu(v)\right\}$ for any cubic Pythagorean fuzzy number.

Definition 2.3.2 in [19] proposed the basic operational laws for CPFNs which are described as follows.

Definition 11 (see [19]). Let $\mathscr{F}_{\mathrm{cp}}=\left\{\left[\mu_{1}^{-}(v), \mu_{1}^{+}(v)\right], \mu_{1}(v)\right\}$ and $\mathscr{F}_{\mathrm{cp}_{2}}=\left\{\left[\mu_{2}^{-}(v), \mu_{2}^{+}(v)\right], \mu_{2}(v)\right\} \in \operatorname{CPFS}(U)$ with $\emptyset>0$. Then, the operational rules are described as

(1) $\mathscr{F}_{\mathrm{cp}_{1}} \oplus \mathscr{F}_{\mathrm{cp}_{2}}=\left\{\left[\sqrt{\left(\mu_{1}^{-}\right)^{2}+\left(\mu_{2}^{-}\right)^{2}-\left(\mu_{1}^{-}\right)^{2} \cdot\left(\mu_{2}^{-}\right)^{2}}\right.\right.$, $\left.\left.\sqrt{\left(\mu_{1}^{+}\right)^{2}+\left(\mu_{2}^{+}\right)^{2}-\left(\mu_{1}^{+}\right)^{2} \cdot\left(\mu_{2}^{+}\right)^{2}}\right], \mu_{1} \cdot \mu_{2}\right\}$

(2) $\mathscr{F}_{\mathrm{cp}_{1}} \otimes \mathscr{F}_{\mathrm{cp}_{2}}=\left\{\left[\mu_{1}^{-} \cdot \mu_{2}^{-}, \mu_{1}^{+} \cdot \mu_{2}^{+}\right]\right.$,

$$
\left.\sqrt{\left(\mu_{1}\right)^{2}+\left(\mu_{2}\right)^{2}-\left(\mu_{1}\right)^{2} \cdot\left(\mu_{2}\right)^{2}}\right\}
$$

(3) $\left(\mathscr{F}_{\mathrm{cp}_{1}}\right)^{\Phi}=\left\{\left[\left(\mu_{1}^{-}\right)^{\Phi},\left(\mu_{1}^{+}\right)^{\Phi}\right], \sqrt{1-\left(1-\mu_{1}^{2}\right)^{\Phi}}\right\}$

(4) $₫ \cdot \mathscr{F}_{\mathrm{cp}_{1}}=\left\{\left[\sqrt{1-\left(1-\left(\mu_{1}^{-}\right)^{2}\right)^{\Phi}}\right.\right.$,

$$
\left.\left.\sqrt{1-\left(1-\left(\mu_{1}^{+}\right)^{2}\right)^{\Phi}}\right],\left(\mu_{1}\right)^{\Phi}\right\}
$$

Fahmi et al. [19] defined Definition 2.3.1 for CPFS and Definition 3 for cubic set which are the same. So, Fahmi et al. [19] presented the invalid definition of cubic Pythagorean fuzzy sets and also presented invalid operational laws for CPFSs.

Now, we presented the valid definition and operational laws for CPFNs are as follows.

Definition 12 (see [17]). A CPFS $\mathscr{F}_{\mathrm{cp}}$ in arbitrary set $U \neq \phi$ has the form

$$
\mathscr{F}=\left\{\left\langle v,\left(\left[\mu^{-}(v), \mu^{+}(v)\right],\left[v^{-}(v), v^{+}(v)\right]\right),(\mu(v), v(v))\right\rangle \mid v \in U\right\},
$$

where $\left[\mu^{-}(v), \mu^{+}(v)\right],\left[v^{-}(v), v^{+}(v)\right] \subseteq[0,1]$ and $\mu(v)$, $v(v) \in[0,1]$ represent the positive membership grade in the form of interval valued Pythagorean fuzzy set and negative membership grade in the form of Pythagorean fuzzy set.

The operational laws for CPFNs are defined as follows.

Definition 13. Let $\quad \mathscr{F}_{\mathrm{cp}_{1}}=\left\{\left(\left[\mu_{1}^{-}(v), \mu_{1}^{+}(v)\right], \quad\left[v_{1}^{-}(v), v_{1}^{+}\right.\right.\right.$ $\left.(v)]),\left(\mu_{1}(v), v_{1}(v)\right)\right\}$ and $\mathscr{F}_{\mathrm{cp}_{2}}=\left\{\left(\left[\mu_{2}^{-}(v), \mu_{2}^{+}(v)\right],\left[v_{2}^{-}(v)\right.\right.\right.$, $\left.\left.\left.v_{2}^{+}(v)\right]\right),\left(\mu_{2}(v), v_{2}(v)\right)\right\} \in \operatorname{CPFS}(U)$ with $₫>0$. Then, the operational rules are described as

$$
\begin{aligned}
\text { (1) } \mathscr{F}_{\mathrm{cp}_{1}} \oplus \mathscr{F}_{\mathrm{cp}_{2}}=\left\{\left(\left[\sqrt{\left(\mu_{1}^{-}\right)^{2}+\left(\mu_{2}^{-}\right)^{2}-\left(\mu_{1}^{-}\right)^{2} \cdot\left(\mu_{2}^{-}\right)^{2}},\right.\right.\right. \\
\left.\left.\sqrt{\left(\mu_{1}^{+}\right)^{2}+\left(\mu_{2}^{+}\right)^{2}-\left(\mu_{1}^{+}\right)^{2} \cdot\left(\mu_{2}^{+}\right)^{2}}\right],\left[v_{1}^{-} \cdot v_{2}^{-}, v_{1}^{+} \cdot v_{2}^{+}\right]\right), \\
\left.\left(\sqrt{\left(\mu_{1}\right)^{2}+\left(\mu_{2}\right)^{2}-\left(\mu_{1}\right)^{2} \cdot\left(\mu_{2}\right)^{2}}, v_{1}(v) \cdot v_{2}(v)\right)\right\} \\
\text { (2) } \mathscr{F}_{\mathrm{cp}_{1}} \otimes \mathscr{F}_{\mathrm{cp}_{2}}=\left\{\left(\left[\mu_{1}^{-} \cdot \mu_{2}^{-}, \mu_{1}^{+} \cdot \mu_{2}^{+}\right],\left[\sqrt{\left(v_{1}^{-}\right)^{2}+\left(v_{2}^{-}\right)^{2}-}\right.\right.\right. \\
\left.\left.\left(v_{1}^{-}\right)^{2} \cdot\left(v_{2}^{-}\right)^{2}, \sqrt{\left(v_{1}^{+}\right)^{2}+\left(v_{2}^{+}\right)^{2}-\left(v_{1}^{+}\right)^{2} \cdot\left(v_{2}^{+}\right)^{2}}\right]\right), \\
\left.\left(\mu_{1}(v) \cdot \mu_{2}(v), \sqrt{\left(v_{1}\right)^{2}+\left(v_{2}\right)^{2}-\left(v_{1}\right)^{2} \cdot\left(v_{2}\right)^{2}}\right)\right\}
\end{aligned}
$$

(3) $\left(\mathscr{F}_{\mathrm{cp}_{1}}\right)^{\Phi}=\left\{\left[\left(\mu_{1}^{-}\right)^{\Phi},\left(\mu_{1}^{+}\right)^{\Phi}\right],\left[\sqrt{1-\left(1-\left(v_{1}^{-}\right)^{2}\right)^{\Phi}}\right.\right.$, $\left.\left.\sqrt{1-\left(1-\left(v_{1}^{+}\right)^{2}\right)^{\Phi}}\right],\left(\left(\mu_{1}\right)^{\Phi}, \sqrt{1-\left(1-\left(v_{1}\right)^{2}\right)^{\Phi}}\right)\right\}$

(4) $₫ \cdot \mathscr{F}_{\mathrm{cp}_{1}}=\left\{\left(\left[\sqrt{1-\left(1-\left(\mu_{1}^{-}\right)^{2}\right)^{\Phi}}\right.\right.\right.$,

$$
\begin{array}{ll}
\left.\sqrt{1-\left(1-\left(\mu_{1}^{+}\right)^{2}\right)^{\Phi}}\right], & \left.\left[\left(v_{1}^{-}\right)^{\Phi},\left(v_{1}^{+}\right)^{\Phi}\right]\right),(\sqrt{1-} \\
\left.\left.\left(1-\left(\mu_{1}\right)^{2}\right)^{\Phi},\left(v_{1}\right)^{\Phi}\right)\right\} &
\end{array}
$$

\section{Counter Section 2.4 of [19]}

This section recalls the discussion of cubic Pythagorean linguistic fuzzy numbers (CPLFN) and their basic operations proposed by Fahmi et al. [19].

Definition 2.4.1 in [19] proposed the concept of CPLFS which is described as follows.

Definition 14 (see [19]). A CPLFS $\mathscr{F}$ in arbitrary set $U \neq \phi$, with $\mathfrak{R}=\left\{\ell_{v_{0}}, \ell_{v_{1}}, \ell_{v_{2}}, \ldots, \ell_{v_{t-1}}\right\}$, is the linguistic term set, where $t>0$ is the odd cardinality, having the form

$$
\mathscr{F}=\left\{\ell_{v},\left\langle\left[\mu^{-}(v), \mu^{+}(v)\right], \mu(v)\right\rangle \mid v \in U\right\},
$$

where $\ell_{v} \in \Re$ and $\left[\mu^{-}(v), \mu^{+}(v)\right] \subseteq[0,1]$ and $\mu(v) \in[0,1]$ represent the positive and negative grades of memberships.

For the whole study, the list of cubic Pythagorean linguistic fuzzy sets are represented by $\operatorname{CPLFS}(U)$. For simplicity, we write $\left\{\ell_{v},\left\langle\left[\mu^{-}(v), \mu^{+}(v)\right], \mu(v)\right\rangle\right\}$ for any cubic Pythagorean linguistic fuzzy number.

Definition 2.4.2 in [19] proposed the basic operational laws for CPLFNs described as follows.

Definition 15 (see [19]). Let $\mathscr{F}_{\mathrm{cp}_{1}}=\left\{\ell_{v_{1}},\left\langle\left[\mu_{1}^{-}(v)\right.\right.\right.$, $\left.\left.\left.\mu_{1}^{+}(v)\right], \mu_{1}(v)\right\rangle\right\}$ and $\mathscr{F}_{\mathrm{cp}_{2}}=\left\{\ell_{v_{2}},\left\langle\left[\mu_{2}^{-}(v), \mu_{2}^{+}(v)\right], \mu_{2}(v)\right\rangle\right\}$ $\in \operatorname{CPLFS}(U)$ with $\Phi>0$. Then, the operational rules are described as

(1) $\mathscr{F}_{\mathrm{cp}_{1}} \oplus \mathscr{F}_{\mathrm{cp}_{2}}=\left\{\ell_{v_{1}+v 2}\right.$,

$$
\begin{aligned}
& \left\langle\left[\sqrt{\left(\mu_{1}^{-}\right)^{2}+\left(\mu_{2}^{-}\right)^{2}-\left(\mu_{1}^{-}\right)^{2} \cdot\left(\mu_{2}^{-}\right)^{2}},\right.\right. \\
& \left.\left.\left.\sqrt{\left(\mu_{1}^{+}\right)^{2}+\left(\mu_{2}^{+}\right)^{2}-\left(\mu_{1}^{+}\right)^{2} \cdot\left(\mu_{2}^{+}\right)^{2}}\right], \mu_{1} \cdot \mu_{2}\right\rangle\right\}
\end{aligned}
$$

(2) $\mathscr{F}_{\mathrm{cp}_{1}} \otimes \mathscr{F}_{\mathrm{cp}_{2}}=\left\{\ell_{v_{1} \times v_{2}},\left\langle\left[\mu_{1}^{-} \cdot \mu_{2}^{-}, \mu_{1}^{+} \cdot \mu_{2}^{+}\right], \sqrt{\left(\mu_{1}\right)^{2}+}\right.\right.$

$$
\left.\left.\left(\mu_{2}\right)^{2}-\left(\mu_{1}\right)^{2} \cdot\left(\mu_{2}\right)^{2}\right\rangle\right\}
$$

(3) $\left(\mathscr{F}_{\mathrm{cp}_{1}}\right)^{\Phi}=\left\{\ell_{v_{1}^{\infty}},\left\langle\left[\left(\mu_{1}^{-}\right)^{\Phi},\left(\mu_{1}^{+}\right)^{\Phi}\right], \sqrt{1-\left(1-\mu_{1}^{2}\right)^{\Phi}}\right\rangle\right\}$

(4) $₫ \cdot \mathscr{F}_{\mathrm{cp}_{1}}=\left\{\ell_{v_{1 \times \Phi}},\left\langle\left[\sqrt{1-\left(1-\left(\mu_{1}^{-}\right)^{2}\right)^{\Phi}}\right.\right.\right.$,

$$
\left.\left.\left.\sqrt{1-\left(1-\left(\mu_{1}^{+}\right)^{2}\right)^{\Phi}}\right],\left(\mu_{1}\right)^{\Phi}\right\rangle\right\}
$$

Fahmi et al. [19] presented Definition 2.4.1 for CPLFS and Definition 3 for cubic set which are the same. So, Fahmi et al. [19] presented the invalid definition of cubic Pythagorean linguistic fuzzy sets and also presented invalid operational laws for CPLFSs.

Now, we presented the valid definition and operational laws for CPLFNs as follows. 
Definition 16. A CLPFS $\mathscr{F}_{\mathrm{cp}}$ in arbitrary set $U \neq \phi$, with $\mathfrak{R}=\left\{\ell_{v_{0}}, \ell_{v_{1}}, \ell_{v_{2}}, \ldots, \ell_{v_{t-1}}\right\}$, is the linguistic term set, where $t>0$ is the odd cardinality, having the form

$$
\mathscr{F}=\left\{\ell_{v},\left(\left(\left[\mu^{-}(v), \mu^{+}(v)\right],\left[v^{-}(v), v^{+}(v)\right]\right),(\mu(v), v(v))\right) \mid v \in U\right\},
$$

where $\ell_{v} \in \mathfrak{R}$ and $\left[\mu^{-}(v), \mu^{+}(v)\right],\left[v^{-}(v), v^{+}(v)\right] \subseteq[0,1]$ and $\mu(v), v(v) \in[0,1]$ represent the positive membership grade in the form of interval-valued Pythagorean fuzzy set and negative membership grade in the form of Pythagorean fuzzy set.

The operational laws for CPLFNs are defined as follows.

Definition 17. Let $\mathscr{F}_{\mathrm{cp}_{1}}=\left\{\ell_{v_{1}},\left(\left(\left[\mu_{1}^{-}(v), \mu_{1}^{+}(v)\right],\left[v_{1}^{-}(v)\right.\right.\right.\right.$, $\left.\left.\left.\left.v_{1}^{+}(v)\right]\right),\left(\mu_{1}(v), v_{1}(v)\right)\right)\right\}$ and $\mathscr{F}_{\mathrm{cp}_{2}}=\left\{\ell_{v_{2}},\left(\left(\left[\mu_{2}^{-}(v), \mu_{2}^{+}(v)\right]\right.\right.\right.$, $\left.\left.\left.\left[v_{2}^{-}(v), v_{2}^{+}(v)\right]\right),\left(\mu_{2}(v), v_{2}(v)\right)\right)\right\} \in \operatorname{CPLFS}(U)$ with $\omega>0$. Then, the operational rules are described as

(1) $\mathscr{F}_{\mathrm{cp}_{1}} \oplus \mathscr{F}_{\mathrm{cp}_{2}}=\left\{\ell_{v_{1}+v_{2}}\right.$, $\left(\left(\left[\sqrt{\left(\mu_{1}^{-}\right)^{2}+\left(\mu_{2}^{-}\right)^{2}-\left(\mu_{1}^{-}\right)^{2} \cdot\left(\mu_{2}^{-}\right)^{2}}\right.\right.\right.$,

$$
\begin{aligned}
& \left.\sqrt{\left(\mu_{1}^{+}\right)^{2}+\left(\mu_{2}^{+}\right)^{2}-\left(\mu_{1}^{+}\right)^{2} \cdot\left(\mu_{2}^{+}\right)^{2}}\right],\left[v_{1}^{-} \cdot v_{2}^{-}, v_{1}^{+} \cdot\right. \\
& \left.\left.\left.\left.v_{2}^{+}\right]\right),\left(\sqrt{\left(\mu_{1}\right)^{2}+\left(\mu_{2}\right)^{2}-\left(\mu_{1}\right)^{2} \cdot\left(\mu_{2}\right)^{2}}, v_{1}(v) \cdot v_{2}(v)\right)\right)\right\}
\end{aligned}
$$

(2) $\mathscr{F}_{\mathrm{cp}_{1}} \otimes \mathscr{F}_{\mathrm{cp}_{2}}=\left\{\ell_{v_{1} \times v_{2}},\left(\left[\mu_{1}^{-} \cdot \mu_{2}^{-}, \mu_{1}^{+} \cdot \mu_{2}^{+}\right]\right.\right.$,

$\left[\sqrt{\left(v_{1}^{-}\right)^{2}+\left(v_{2}^{-}\right)^{2}-\left(v_{1}^{-}\right)^{2} \cdot\left(v_{2}^{-}\right)^{2}}\right.$, $\left.\left.\sqrt{\left(v_{1}^{+}\right)^{2}+\left(v_{2}^{+}\right)^{2}-\left(v_{1}^{+}\right)^{2} \cdot\left(v_{2}^{+}\right)^{2}}\right]\right),\left(\mu_{1}(v) \cdot \mu_{2}(v)\right.$, $\left.\left.\sqrt{\left(v_{1}\right)^{2}+\left(v_{2}\right)^{2}-\left(v_{1}\right)^{2} \cdot\left(v_{2}\right)^{2}}\right)\right\}$

(3) $\left(\mathscr{F}_{\mathrm{cp}_{1}}\right)^{\varpi}=\left\{\ell_{v_{1}^{\oplus}},\left(\left(\left[\left(\mu_{1}^{-}\right)^{\varpi},\left(\mu_{1}^{+}\right)^{Ф}\right]\right.\right.\right.$, $\left.\left[\sqrt{1-\left(1-\left(v_{1}^{-}\right)^{2}\right)^{\Phi}}, \sqrt{1-\left(1-\left(v_{1}^{+}\right)^{2}\right)^{\Phi}}\right]\right)$, $\left.\left.\left.\left(\left(\mu_{1}\right)^{\oplus}, \sqrt{1-(1-}\left(v_{1}\right)^{2}\right)^{\Phi}\right)\right)\right\}$

(4) $\varpi \cdot \mathscr{F}_{\mathrm{cp}_{1}}=\left\{\ell_{v_{1} \times \omega},\left(\left(\left[\sqrt{1-\left(1-\left(\mu_{1}^{-}\right)^{2}\right)^{\Phi}}\right.\right.\right.\right.$,

$\left.\sqrt{1-\left(1-\left(\mu_{1}^{+}\right)^{2}\right)^{\varpi}}\right]$, $\left.\left[\left(v_{1}^{-}\right)^{\oplus},\left(v_{1}^{+}\right)^{\oplus}\right]\right)$, $\left.\left.\left(\sqrt{1-\left(1-\left(\mu_{1}\right)^{2}\right)^{\Phi}},\left(v_{1}\right)^{\Phi}\right)\right)\right\}$

Definition 18. Let

$$
\begin{aligned}
\mathscr{F}_{\mathrm{cp}_{i}} & =\left\{\ell_{v_{i}},\left(\left[\mu_{i}^{-}, \mu_{i}^{+}\right],\left[v_{i}^{-}, v_{i}^{+}\right]\right),\left(\mu_{i}, v_{i}\right)\right\}(i \in \mathbb{N}) \in \operatorname{CPLFS}(U) \cdot \mathcal{S}_{\gamma}\left(\mathscr{F}_{\mathrm{cp}_{(1)}}\right) \\
& =\frac{\left(\left(\mu_{1}^{+}\right)^{2}-\left(v_{1}^{+}\right)^{2}\right)+\left(\left(\mu_{1}\right)^{2}-\left(v_{1}\right)^{2}\right) \times v_{1}}{4} \\
\mathcal{S}_{\gamma}\left(\mathscr{F}_{\mathrm{cp}_{(2)}}\right) & =\frac{\left(\left(\mu_{2}^{+}\right)^{2}-\left(v_{2}^{+}\right)^{2}\right)+\left(\left(\mu_{2}\right)^{2}-\left(v_{2}\right)^{2}\right) \times v_{2}}{4}
\end{aligned}
$$

be the score values of CPLFNs. Also,

$$
\begin{aligned}
& \mathscr{A}_{y}\left(\mathscr{F}_{\mathrm{cp}_{(1)}}\right)=\frac{\left(\left(\mu_{1}^{+}\right)^{2}+\left(v_{1}^{+}\right)^{2}\right)+\left(\left(\mu_{1}\right)^{2}+\left(v_{1}\right)^{2}\right) \times v_{1}}{4}, \\
& \mathscr{A}_{y}\left(\mathscr{F}_{\mathrm{cp}_{(2)}}\right)=\frac{\left(\left(\mu_{2}^{+}\right)^{2}+\left(v_{2}^{+}\right)^{2}\right)+\left(\left(\mu_{2}\right)^{2}+\left(v_{2}\right)^{2}\right) \times v_{2}}{4},
\end{aligned}
$$

are the accuracy values of CPLFNs. If

(a) $\mathcal{S}_{\gamma}\left(\mathscr{F}_{\mathrm{cp}_{(1)}}\right)<\mathcal{S}_{\gamma}\left(\mathscr{F}_{\mathrm{cp}_{(2)}}\right) \Rightarrow \mathscr{F}_{\mathrm{cp}_{(1)}}<\mathscr{F}_{\mathrm{cp}_{(2)}}$

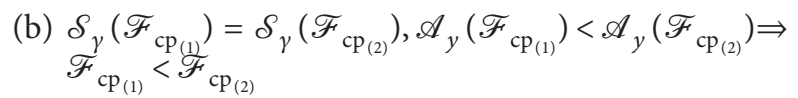

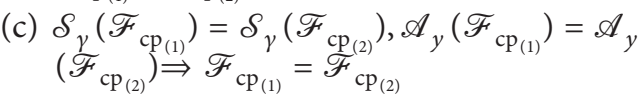

\section{Counter Section 3 of [19]}

This section recalls the discussion of linguistic aggregation operators (AO) for CPLFN and their basic properties proposed by Fahmi et al. [19].

5.1. Weighted Averaging. Definition 3.1.1 and Theorem 3.1.2 in [19] proposed the weighted averaging operator using defined operational rules described as follows.

Definition 19 (see [19]). Let $\mathscr{F}_{\mathrm{cp}_{i}}=\left\{\ell_{v_{i}},\left\langle\left[\mu_{i}^{-}(v), \mu_{i}^{+}(v)\right], \mu_{i}\right.\right.$ $(v)\rangle\} ;(i \in \mathbb{N}) \in \operatorname{CPLFS}(U)$. Then, the CPLFWA operator is described as follows:

$\operatorname{CPLFWA}\left(\mathscr{F}_{\mathrm{cp}_{1},}, \mathscr{F}_{\mathrm{cp}_{2}}, \ldots, \mathscr{F}_{\mathrm{cp}_{n}}\right)=\left(\frac{1}{n !}\left(\sum_{\theta \in S_{n}}\left(\prod_{i=1}^{n} \mathscr{F}_{c p_{\theta(i)}}\right)\right)\right)$ 
where $\theta(i)(i \in \mathbb{N})$ represents any permutation and $S_{n}$ represents the total numbers of elements.
Theorem 1 (see [19]). Let $\mathscr{F}_{c p_{i}}=\left\{\ell_{v_{i}},\left\langle\left[\mu_{i}^{-}(v), \mu_{i}^{+}(v)\right]\right.\right.$, $\left.\left.\mu_{i}(v)\right\rangle\right\} ;(i \in \mathbb{N}) \in \operatorname{CPLFS}(U)$. Then, using defined operational laws, CPLFWA operator is obtained as

$$
\begin{aligned}
& \operatorname{CPLFWA}\left(\mathscr{F}_{c p_{1}}, \mathscr{F}_{c p_{2}}, \ldots, \mathscr{F}_{c p_{n}}\right) \\
& =\left\{\ell\left(\sum_{i=1}^{n} v_{i} / n\right),\left(\left[\sqrt{1-\left(1-\prod_{i=1}^{n}\left(\mu_{i}^{-}\right)^{2}\right)^{(1 / n !)}}, \sqrt{1-\left(1-\prod_{i=1}^{n}\left(\mu_{i}^{+}\right)^{2}\right)^{(1 / n !)}}\right],\left(\prod_{i=1}^{n}\left(\mu_{i}^{2}\right)\right)^{(1 / n !)}\right)\right\} .
\end{aligned}
$$

Definition 3.2.1 and Theorem 3.2.2 in [19] proposed the generalized weighted averaging operator using defined operational rules described as follows.

Definition 20 (see [19]). Let $\mathscr{F}_{\mathrm{cp}_{i}}=\left\{\ell_{v_{i}},\left\langle\left[\mu_{i}^{-}(v), \mu_{i}^{+}(v)\right]\right.\right.$, $\left.\left.\mu_{i}(v)\right\rangle\right\} ;(i \in \mathbb{N}) \in \operatorname{CPLFS}(U)$. Then, the CGPLFWA operator is described as follows:

$$
\begin{aligned}
& \operatorname{CGPLFWA}\left(\mathscr{F}_{\mathrm{cp}_{1}}, \mathscr{F}_{\mathrm{cp}_{2}}, \ldots, \mathscr{F}_{\mathrm{cp}_{n}}\right) \\
& =\left(\frac{1}{n !}\left(\sum_{\theta \in S_{n}}\left(\prod_{i=1}^{n} \mathscr{F}_{\mathrm{cp}_{\theta(i)}}\right)\right)^{(1 / \lambda)}\right),
\end{aligned}
$$

where $\theta(i)(i \in \mathbb{N})$ represents any permutation and $S_{n}$ represents the total numbers of elements.

Theorem 2 (see [19]). Let $\mathscr{F}_{c p_{i}}=\left\{\ell_{v_{i}},\left\langle\left[\mu_{i}^{-}(v), \mu_{i}^{+}(v)\right]\right.\right.$, $\left.\left.\mu_{i}(v)\right\rangle\right\} ;(i \in \mathbb{N}) \in \operatorname{CGPLFS}(U)$. Then, using defined operational laws, the CGPLFWA operator is obtained as

$$
\begin{aligned}
& \operatorname{CGPLFWA}\left(\mathscr{F}_{\mathrm{cp}_{1}}, \mathscr{F}_{\mathrm{cp}_{2}}, \ldots, \mathscr{F}_{\mathrm{cp}_{n}}\right)
\end{aligned}
$$

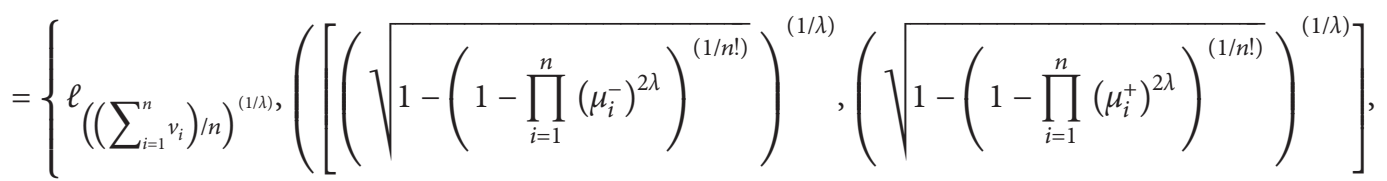

$$
\begin{aligned}
& \sqrt{\left.\left.1-\left(1-\prod_{i=1}^{n}\left(1-\left(1-\mu_{i}^{2}\right)^{\lambda}\right)^{(1 / n !)}\right)^{(1 / \lambda)}\right)\right\}} \text {. }
\end{aligned}
$$

5.2. Weighted Geometric. Definition 3.3.1 and Theorem 3.3.2 in [19] proposed the weighted geometric operator using defined operational rules described as follows.

Definition 21 (see [19]). Let $\mathscr{F}_{\mathrm{cp}_{i}}=\left\{\ell_{v_{i}},\left\langle\left[\mu_{i}^{-}(v), \mu_{i}^{+}(v)\right]\right.\right.$, $\left.\left.\mu_{i}(v)\right\rangle\right\} ;(i \in \mathbb{N}) \in \operatorname{CPLFS}(U)$. Then, the CPLFWG operator is described as follows:

$$
\begin{array}{r}
\operatorname{CPLFWG}\left(\mathscr{F}_{\mathrm{cp}_{1}}, \mathscr{F}_{\mathrm{cp}_{2}}, \ldots, \mathscr{F}_{\mathrm{cp}_{n}}\right) \\
=\left(\frac{1}{n !}\left(\sum_{\theta \in S_{n}}\left(\prod_{i=1}^{n} \mathscr{F}_{{ }_{\mathrm{cp}_{\theta(i)}}}\right)\right)\right),
\end{array}
$$

where $\theta(i)(i \in \mathbb{N})$ represents any permutation and $S_{n}$ represents the total numbers of elements. 
Theorem 3 (see [19]). Let $\mathscr{F}_{c p_{i}}=\left\{\ell_{v_{i}},\left\langle\left[\mu_{i}^{-}(v), \mu_{i}^{+}(v)\right]\right.\right.$, $\left.\left.\mu_{i}(v)\right\rangle\right\} ;(i \in \mathbb{N}) \in C P L F S(U)$. Then, using defined operational laws, the CPLFWG operator is obtained as

$$
\begin{aligned}
& \operatorname{CPLFWG}\left(\mathscr{F}_{\mathrm{cp}_{1}}, \mathscr{F}_{\mathrm{cp}_{2}}, \ldots, \mathscr{F}_{\mathrm{cp}_{n}}\right) \\
& \quad=\left\{\ell_{\left(\sum_{i=1}^{n} v_{i}^{(1 / n)} / n\right)},\left(\left[\left(\sqrt{1-\left(1-\prod_{i=1}^{n}\left(\mu_{i}^{-}\right)^{2 \lambda}\right)^{(1 / n !)}}\right)^{(1 / \lambda)},\left(\sqrt{1-\left(1-\prod_{i=1}^{n}\left(\mu_{i}^{+}\right)^{2 \lambda}\right)^{(1 / n !)}}\right) \sqrt{1-\prod_{i=1}^{n}\left(1-\mu_{i}^{2}\right)^{(1 / n !)}}\right)\right\} .\right.
\end{aligned}
$$

\section{Updated Linguistic Cubic Pythagorean Fuzzy AO}

In this section, utilizing valid Definition 16 of LCPFS and operational laws (Definition 17), we establish the updated operational laws to aggregate the uncertain data in the form of linguistic cubic Pythagorean fuzzy environment.

\subsection{Updated Weighted Averaging AO}

Definition 22. Let $\mathscr{F}_{\mathrm{cp}_{i}}=\left\{\ell_{v_{i}},\left(\left(\left[\mu_{i}^{-}(v), \mu_{i}^{+}(v)\right],\left[v_{i}^{-}(v)\right.\right.\right.\right.$, $\left.\left.\left.\left.v_{i}^{+}(v)\right]\right),\left(\mu_{i}(v), v_{i}(v)\right)\right)\right\} ;(i \in \mathbb{N}) \in \operatorname{CPLFS}(U)$. Then, the CPLFWA operator is described as follows:

$$
\begin{aligned}
& \operatorname{CPLFWA}\left(\mathscr{F}_{\mathrm{cp}_{1}}, \mathscr{F}_{\mathrm{cp}_{2}}, \ldots, \mathscr{F}_{\mathrm{cp}_{n}}\right) \\
& =\rho_{1} \mathscr{F}_{c p_{1}} \oplus \rho_{2} \mathscr{F}_{\mathrm{cp}_{2}} \oplus \cdots \oplus \rho_{n} \mathscr{F}_{\mathrm{cp}_{n}} \\
& =\sum_{\natural=1}^{n} \rho_{\natural} \mathscr{F}_{\mathrm{cp}_{\natural}},
\end{aligned}
$$

where the weights $\left(\rho_{1}, \rho_{2}, \ldots, \rho_{\natural}\right)$ of $\mathscr{F}_{\natural}$ have $\rho_{\natural} \geq 0$ and $\sum_{\natural=1}^{n} \rho_{\natural}=1$.

Theorem 4. Let $\mathscr{F}_{c p_{i}}=\left\{\ell_{v_{i}},\left(\left(\left[\mu_{i}^{-}(v), \mu_{i}^{+}(v)\right],\left[v_{i}^{-}(v), v_{i}^{+}(v)\right]\right)\right.\right.$, $\left.\left.\left(\mu_{i}(v), v_{i}(v)\right)\right)\right\} ;(i \in \mathbb{N}) \in C P L F S(U)$. Then, using defined operational laws 17, CPLFWA operator is obtained as

$$
\begin{aligned}
& \operatorname{CPLFWA}\left(\mathscr{F}_{\mathrm{cp}_{1}}, \mathscr{F}_{\mathrm{cp}_{2}}, \ldots, \mathscr{F}_{\mathrm{cp}_{n}}\right) \\
& =\sum_{\natural=1}^{n} \rho_{\natural} \mathscr{F}_{\mathrm{cp}_{\natural}}
\end{aligned}
$$

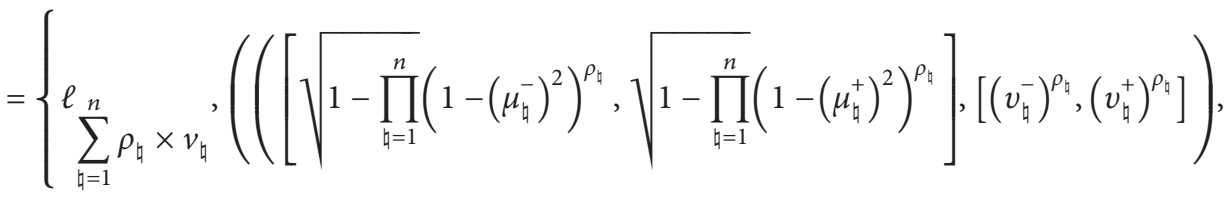

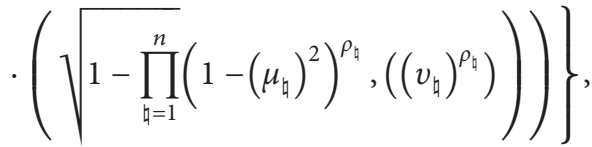

where the weights $\left(\rho_{1}, \rho_{2}, \ldots, \rho_{\natural}\right)$ of $\mathscr{F}_{\natural}$ have $\rho_{\natural} \geq 0$ and $\sum_{\natural=1}^{n} \rho_{\natural}=1$.

6.2. Updated Weighted Geometric AO
Definition 23. Let $\mathscr{F}_{\mathrm{cp}_{i}}=\left\{\ell_{v_{i}},\left(\left(\left[\mu_{i}^{-}(v), \mu_{i}^{+}(v)\right],\left[v_{i}^{-}\right.\right.\right.\right.$ $\left.\left.\left.\left.(v), v_{i}^{+}(v)\right]\right),\left(\mu_{i}(v), v_{i}(v)\right)\right)\right\} ;(i \in \mathbb{N}) \in \operatorname{CPLFS}(U)$. Then, the CPLFWG operator is described as follows:

$$
\begin{aligned}
\operatorname{CPLFWG}\left(\mathscr{F}_{\mathrm{cp}_{1}}, \mathscr{F}_{\mathrm{cp}_{2}}, \ldots, \mathscr{F}_{\mathrm{cp}_{n}}\right) & =\left(\mathscr{F}_{\mathrm{cp}_{1}}\right)^{\rho_{1}} \otimes\left(\mathscr{F}_{\mathrm{cp}_{2}}\right)^{\rho_{2}} \otimes \cdots \otimes\left(\mathscr{F}_{\mathrm{cp}_{n}}\right)^{\rho_{n}} \\
& =\prod_{\natural=1}^{n}\left(\mathscr{F}_{\mathrm{cp}_{\natural}}\right)^{\rho_{\natural}},
\end{aligned}
$$


where the weights $\left(\rho_{1}, \rho_{2}, \ldots, \rho_{\natural}\right)$ of $\mathscr{F}_{\natural}$ have $\rho_{\natural} \geq 0$ and $\sum_{\natural=1}^{n} \rho_{\natural}=1$.
Theorem 5. Let $\mathscr{F}_{c p_{i}}=\left\{\ell_{v_{i}},\left(\left(\left[\mu_{i}^{-}(v), \mu_{i}^{+}(v)\right],\left[v_{i}^{-}(v)\right.\right.\right.\right.$, $\left.\left.\left.\left.v_{i}^{+}(v)\right]\right), \quad\left(\mu_{i}(v), v_{i}(v)\right)\right)\right\} ;(i \in \mathbb{N}) \in \operatorname{CPLFS}(U)$. Then, using defined operational laws 17, the CPLFWG operator is obtained as

$$
\begin{aligned}
& \operatorname{CPLFWG}\left(\mathscr{F}_{\mathrm{cp}_{1}}, \mathscr{F}_{\mathrm{cp}_{2}}, \ldots, \mathscr{F} \%_{\mathrm{cp}_{n}}\right) \\
& =\prod_{\natural=1}^{n}\left(\mathscr{F}_{c p_{\natural}}\right)^{\rho_{\natural}}
\end{aligned}
$$

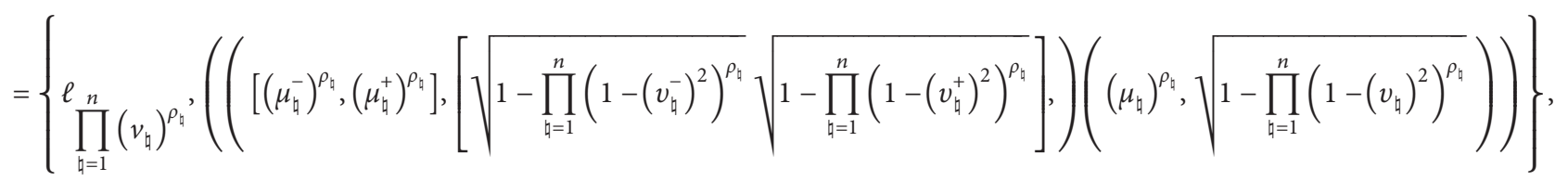

where the weights $\left(\rho_{1}, \rho_{2}, \ldots, \rho_{\natural}\right)$ of $\mathscr{F}_{\natural}$ having $\rho_{\natural} \geq 0$ and $\sum_{\natural=1}^{n} \rho_{\natural}=1$.

\section{Countersections 3.4 and 3.5 of [19]}

This section recalls the discussion of linguistic MSM aggregation operators (AO) for CPLFN and their basic properties proposed by Fahmi et al. [19].

Definition 3.4.1 and Theorem 3.4.2 in [19] proposed the MSM operator using defined operational rules (Definition 15) described as follows.

Definition 24 (see [19]). Let $\mathscr{F}_{\mathrm{cp}_{i}}=\left\{\ell_{v_{i}},\left\langle\left[\mu_{i}^{-}(v), \mu_{i}^{+}(v)\right]\right.\right.$, $\left.\left.\mu_{i}(v)\right\rangle\right\} ;(i \in \mathbb{N}) \in \operatorname{CPLFS}(U)$ and $\left(\rho_{1}, \rho_{2}, \ldots, \rho_{\natural}\right)^{T}$ is weight vector having $\rho_{\natural} \geq 0$ and $\sum_{\natural=1}^{n} \rho_{\natural}=1$. Then, the CPLFMSM operator is described as follows:

$$
\begin{aligned}
& \operatorname{CPLFMSM}\left(\mathscr{F}_{\mathrm{cp}_{1}}, \mathscr{F}_{\mathrm{cp}_{2}}, \ldots, \mathscr{F}_{\mathrm{cp}_{n}}\right) \\
& =\left(\frac{1}{n !}\left(\sum_{\theta \in S_{n}}\left(\prod_{i=1}^{n} \mathscr{F}_{c p_{\theta(i)}}\right)\right)^{(1 / \lambda)}\right),
\end{aligned}
$$

where $\theta(i)(i \in \mathbb{N})$ represents any permutation and $S_{n}$ represents the total numbers of elements.

Theorem 6 (see [19]). Let $\mathscr{F}_{c p_{i}}=\left\{\ell_{v_{i}},\left\langle\left[\mu_{i}^{-}(v), \mu_{i}^{+}(v)\right]\right.\right.$, $\left.\left.\mu_{i}(v)\right\rangle\right\} ;(i \in \mathbb{N}) \in \operatorname{CPLFS}(U)$. Then, using defined operational laws, the CPLFMSM operator is obtained as

$$
\begin{aligned}
& \operatorname{CPLFMSM}\left(\mathscr{F}_{\mathrm{cp}_{1}}, \mathscr{F}_{\mathrm{cp}_{2}}, \ldots, \mathscr{F}_{\mathrm{cp}_{n}}\right) \\
& =\ell\left(\left(1 / \mathbb{C}_{n}^{k}\right)\left(\sum_{1 \leq i_{1} \leq i_{2} \leq \ldots \leq i_{k} \leq n} \prod_{j=1}^{k} h_{i_{j}}\right)\right)^{(1 / \lambda),} \\
& \cdot\left(\left[\sqrt{1-\left(\prod_{1 \leq i_{1} \leq i_{2} \leq \cdots \leq i_{k} \leq n}\left(1-\prod_{i=1}^{n}\left(\mu_{i}^{-}\right)^{2}\right)^{\left(1 / \mathbb{C}_{n}^{k}\right)}\right)^{(1 / k)}}, \sqrt{1-\left(\prod_{1 \leq i_{1} \leq i_{2} \leq \cdots \leq i_{k} \leq n}\left(1-\prod_{i=1}^{n}\left(\mu_{i}^{+}\right)^{2}\right)^{\left(1 / \mathbb{C}_{n}^{k}\right)}\right)^{(1 / k)}}\right],\right. \\
& \left.\left.\sqrt{1-\left(1-\left(\prod_{1 \leq i_{1} \leq i_{2} \leq \cdots \leq i_{k} \leq n}\left(1-\prod_{i=1}^{n}\left(1-\mu_{i}^{2}\right)^{\left(1 / \mathbb{C}_{n}^{k}\right)}\right)\right)^{(1 / k)}\right)}\right)\right\} \text {. }
\end{aligned}
$$

Definition 3.5.1 and Theorem 3.5.2 in [19] proposed the weighted MSM operator using defined operational rules (Definition 15) described as follows.

Definition 25 (see [19]). Let $\mathscr{F}_{\mathrm{cp}_{i}}=\left\{\ell_{v_{i}},\left\langle\left[\mu_{i}^{-}(v), \mu_{i}^{+}(v)\right]\right.\right.$, $\left.\left.\mu_{i}(v)\right\rangle\right\} ;(i \in \mathbb{N}) \in \operatorname{CPLFS}(U)$ and $\left(\rho_{1}, \rho_{2}, \ldots, \rho_{n}\right)^{T}$ is weight vector having $\rho_{\natural} \geq 0$ and $\sum_{\natural=1}^{n} \rho_{\natural}=1$. Then, the CPLFWMSM operator is described as follows:

$$
\begin{array}{r}
\operatorname{CPLFWMSM}\left(\mathscr{F}_{\mathrm{cp}_{1}}, \mathscr{F}_{\mathrm{cp}_{2}}, \ldots, \mathscr{F}_{\mathrm{cp}_{n}}\right) \\
=\left(\frac{1}{n !}\left(\sum_{\theta \in S_{n}}\left(\prod_{i=1}^{n} \mathscr{F}_{\mathrm{cp}_{\theta(i)}}\right)\right)^{(1 / \lambda)}\right),
\end{array}
$$

where $\theta(i)(i \in \mathbb{N})$ represents any permutation and $S_{n}$ represents the total numbers of elements. 
Theorem 7 (see [19]). Let $\mathscr{F}_{c p_{i}}=\left\{\ell_{v_{i}},\left\langle\left[\mu_{i}^{-}(v), \mu_{i}^{+}(v)\right]\right.\right.$, $\left.\left.\mu_{i}(v)\right\rangle\right\} ;(i \in \mathbb{N}) \in C P L F S(U)$. Then, using defined operational laws, CPLFWMSM operator is obtained as

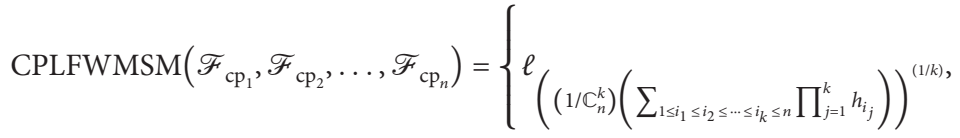

$$
\begin{aligned}
& \cdot\left[\sqrt{\left(1-\prod_{1 \leq i_{1} \leq i_{2} \leq \cdots \leq i_{k} \leq n}\left(1-\prod_{i=1}^{n}\left(1-\left(\mu_{i}^{-}\right)^{2}\right)^{\rho_{i}}\right)^{\left(1 / \mathbb{C}_{n}^{k}\right)}\right)^{(1 / k)}}, \sqrt{\left(1-\prod_{1 \leq i_{1} \leq i_{2} \leq \cdots \leq i_{k} \leq n}\left(1-\prod_{i=1}^{n}\left(1-\left(\mu_{i}^{+}\right)^{2}\right)^{\rho_{i}}\right)^{\left(1 / \mathbb{C}_{n}^{k}\right)}\right)^{(1 / k)}}\right] \\
& \left.\sqrt{1-\left(1-\left(\prod_{1 \leq i_{1} \leq i_{2} \leq \cdots \leq i_{k} \leq n}\left(1-\prod_{i=1}^{n}\left(1-\left(\mu_{i}^{2}\right)^{\rho_{i}}\right)^{\left(1 / \mathbb{C}_{n}^{k}\right)}\right)\right)^{(1 / k)}\right)}\right\} \text {. }
\end{aligned}
$$

\section{Updated Linguistic Cubic Pythagorean Fuzzy MSM AO}

In this section, utilizing valid Definition 16 of LCPFS and operational laws (Definition 17), we establish the updated linguistic Maclaurin symmetric mean AO to aggregate the uncertain data in the form of linguistic cubic Pythagorean fuzzy environment.

Definition 26. Let $\mathscr{F}_{\mathrm{cp}_{i}}=\left\{\ell_{v_{i}},\left(\left(\left[\mu_{i}^{-}(v), \mu_{i}^{+}(v)\right],\left[v_{i}^{-}(v)\right.\right.\right.\right.$, $\left.\left.\left.\left.v_{i}^{+}(v)\right]\right), \quad\left(\mu_{i}(v), v_{i}(v)\right)\right)\right\} ;(i \in \mathbb{N}) \in \operatorname{CPLFS}(U)$. Then, the CPLFMSM operator is described as follows:

$$
\begin{aligned}
& \operatorname{CPLFMSM}\left(\mathscr{F}_{\mathrm{cp}_{1}}, \mathscr{F}_{\mathrm{cp}_{2}}, \ldots, \mathscr{F}_{\mathrm{cp}_{n}}\right) \\
& =\left(\sum_{1 \leq i_{1} \leq i_{2} \leq \cdots \leq i_{k} \leq n} \frac{\prod_{j=1}^{k} \mathscr{F}_{\mathrm{cp}_{i j}}}{\mathbb{C}_{n}^{k}}\right)^{(1 / k)},
\end{aligned}
$$

where $\mathbb{C}_{n}^{k}$ is the binomial coefficient and $\left(i_{1}, i_{2}, \ldots, i_{k}\right)$ traversal of all the k-tuple combination of $(1,2, \ldots, n)$.

Theorem 8. Let $\mathscr{F}_{c p_{i}}=\left\{\ell_{v_{i}},\left(\left(\left[\mu_{i}^{-}(v), \mu_{i}^{+}(v)\right],\left[v_{i}^{-}(v)\right.\right.\right.\right.$, $\left.\left.\left.\left.v_{i}^{+}(v)\right]\right),\left(\mu_{i}(v), v_{i}(v)\right)\right)\right\} ;(i \in \mathbb{N}) \in \operatorname{CPLFS}(U)$. Then, using defined operational laws 17, the CPLFMSM operator is obtained as

$$
\begin{aligned}
& \operatorname{CPLFMSM}\left(\mathscr{F}_{\mathrm{cp}_{1}}, \mathscr{F}_{\mathrm{cp}_{2}}, \ldots, \mathscr{F}_{\mathrm{cp}_{n}}\right)=\left(\frac{\sum_{1 \leq i_{1} \leq i_{2} \leq \cdots \leq i_{k} \leq n} \prod_{j=1}^{k} \mathscr{F}_{\mathrm{cp}_{i j}}}{\mathbb{C}_{n}^{k}}\right)^{(1 / k)} \\
& =\left\{\ell^{\ell}\left(\sum_{1 \leq i_{1} \leq i_{2} \leq \cdots \leq i_{k} \leq n} \prod_{j=1}^{k} \mathscr{F}_{c p_{i_{j}}} / \mathbb{C}_{n}^{k}\right)^{(1 / k),}\right.
\end{aligned}
$$

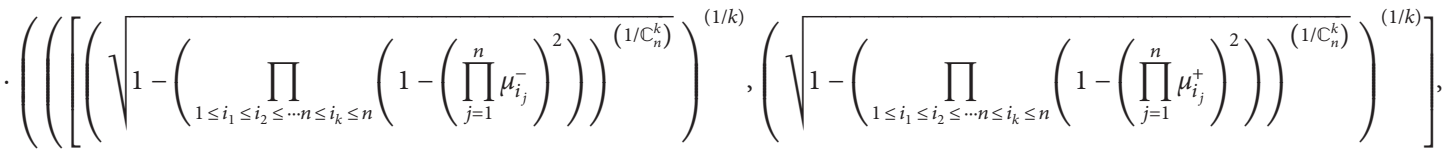

$$
\begin{aligned}
& \left.\cdot\left[\sqrt{1-\left(1-\left(\prod_{1 \leq i_{1} \leq i_{2} \leq \cdots \leq i_{k} \leq n}\left(1-\prod_{j=1}^{n}\left(1-\left(v_{i_{j}}^{-}\right)^{2}\right)\right)\right)^{\left(1 / \mathbb{C}_{n}^{k}\right)}\right)^{(1 / k)}}, \sqrt{1-\left(1-\left(\prod_{1 \leq i_{1} \leq i_{2} \leq \cdots \leq i_{k} \leq n}\left(1-\prod_{j=1}^{n}\left(1-\left(v_{i_{j}}^{+}\right)^{2}\right)\right)\right)^{\left(1 / \mathbb{C}_{n}^{k}\right)}\right)^{(1 / k)}}\right]\right) \\
& \left.\left.\cdot\left(\sqrt{1-\left(\prod_{1 \leq i_{1} \leq i_{2} \leq \cdots n \leq i_{k} \leq n}\left(1-\left(\prod_{j=1}^{n} \mu_{i_{j}}\right)^{2}\right)\right)^{\left(1 / \mathbb{C}_{n}^{k}\right)}}\right)^{(1 / k)}, \sqrt{1-\left(1-\left(\prod_{1 \leq i_{1} \leq i_{2} \leq \cdots \leq i_{k} \leq n}\left(1-\prod_{j=1}^{n}\left(1-\left(v_{i_{j}}\right)^{2}\right)\right)\right)^{\left(1 / \mathbb{C}_{n}^{k}\right)}\right)^{(1 / k)}}\right)\right\} .
\end{aligned}
$$


Updated weighted MSM AO is defined as follows.

Definition 27. Let $\mathscr{F}_{\mathrm{cp}_{i}}=\left\{\ell_{v_{i}},\left(\left(\left[\mu_{i}^{-}(v), \mu_{i}^{+}(v)\right],\left[v_{i}^{-}(v)\right.\right.\right.\right.$, $\left.\left.\left.\left.v_{i}^{+}(v)\right]\right),\left(\mu_{i}(v), v_{i}(v)\right)\right)\right\} ;(i \in \mathbb{N}) \in \operatorname{CPLFS}(U) \quad$ and $\left(\rho_{1}, \rho_{2}, \ldots, \rho_{n}\right)^{T}$ is weight vector having $\rho_{\natural} \geq 0$ and $\sum_{\natural=1}^{n} \rho_{\natural}=1$. Then, the CPLFWMSM operator is described as follows:

$$
\begin{aligned}
& \operatorname{CPLFWMSM}\left(\mathscr{F}_{\mathrm{cp}_{1}}, \mathscr{F}_{\mathrm{cp}_{2}}, \ldots, \mathscr{F}_{\mathrm{cp}_{n}}\right) \\
& =\left(\frac{\sum_{1 \leq i_{1} \leq i_{2} \leq \cdots \leq i_{k} \leq n}\left(\prod_{j=1}^{k}\left(\mathscr{F}_{c p_{i_{j}}}\right)^{\rho_{i_{j}}}\right)}{\mathbb{C}_{n}^{k}}\right)^{(1 / k)},
\end{aligned}
$$

where $\mathbb{C}_{n}^{k}$ is the binomial coefficient and $\left(i_{1}, i_{2}, \ldots, i_{k}\right)$ traversal of all the k-tuple combination of $(1,2, \ldots, n)$.

Theorem 9. Let $\mathscr{F}_{c p_{i}}=\left\{\ell_{v_{i}},\left(\left(\left[\mu_{i}^{-}(v), \mu_{i}^{+}(v)\right],\left[v_{i}^{-}(v)\right.\right.\right.\right.$, $\left.\left.\left.\left.v_{i}^{+}(v)\right]\right),\left(\mu_{i}(v), v_{i}(v)\right)\right)\right\} ;(i \in \mathbb{N}) \in \operatorname{CPLFS}(U)$. Then, using defined operational laws 17, the CPLFWMSM operator is obtained as

$$
\begin{aligned}
& \operatorname{CPLFWMSM}\left(\mathscr{F}_{\mathrm{cp}_{1}}, \mathscr{F}_{\mathrm{cp}_{2}}, \ldots, \mathscr{F}_{\mathrm{cp}_{n}}\right)=\left(\frac{\sum_{1 \leq i_{1} \leq i_{2} \leq \cdots \leq i_{k} \leq n}\left(\prod_{j=1}^{k}\left(\mathscr{F}_{\mathrm{cp}_{i_{j}}}\right)^{\rho_{i_{j}}}\right)}{\mathbb{C}_{n}^{k}}\right)^{(1 / k)} \\
& =\left\{\ell\left(\sum_{1 \leq i_{1} \leq i_{2} \leq \ldots \leq i_{k} \leq n}\left(\prod_{j=1}^{k}\left(\mathscr{F}_{c p_{i_{j}}}\right)^{p_{i_{j}}}\right) / \mathbb{C}_{n}^{k}\right)^{(1 / k),}\right. \\
& \cdot\left(\left[\left(\sqrt{1-\left(\prod_{1 \leq i_{1} \leq i_{2} \leq \ldots \leq i_{k} \leq n} 1-\left(\prod_{j=1}^{n}\left(\mu_{i_{j}}^{-}\right)^{\rho_{i_{j}}}\right)^{2}\right)^{\left(1 / \mathbb{C}_{n}^{k}\right)}}\right)^{(1 / k)},\right.\right. \\
& \left.\left(\sqrt{1-\left(\prod_{1 \leq i_{1} \leq i_{2} \leq \ldots \leq i_{k} \leq n} 1-\left(\prod_{j=1}^{n}\left(\mu_{i_{j}}^{+}\right)^{\rho_{i_{j}}}\right)^{2}\right)^{\left(1 / \mathbb{C}_{n}^{k}\right)}}\right)^{(1 / k)}\right] \\
& \sqrt{1-\left(1-\left(\prod_{1 \leq i_{1} \leq i_{2} \leq \ldots \leq i_{k} \leq n}\left(1-\prod_{j=1}^{n}\left(1-\left(v_{i_{j}}^{-}\right)^{2}\right)^{\rho_{i_{j}}}\right)\right)^{\left(1 / \mathbb{C}_{n}^{k}\right)}\right)^{(1 / k)}}, \\
& \sqrt{1-\left(1-\left(\prod_{1 \leq i_{1} \leq i_{2} \leq \ldots \leq i_{k} \leq n}\left(1-\prod_{j=1}^{n}\left(1-\left(v_{i_{j}}^{+}\right)^{2}\right)^{\rho_{i_{j}}}\right)\right)^{\left(1 / \mathbb{C}_{n}^{k}\right)}\right)^{(1 / k)}}, \\
& \left(\sqrt{1-\left(\prod_{1 \leq i_{1} \leq i_{2} \leq \ldots \leq i_{k} \leq n} 1-\left(\prod_{j=1}^{n}\left(\mu_{i_{j}}\right)^{\rho_{i_{j}}}\right)^{2}\right)^{\left(1 / \mathbb{C}_{n}^{k}\right)}}\right)^{(1 / k)} \\
& \sqrt{\left.\left.1-\left(1-\left(\prod_{1 \leq i_{1} \leq i_{2} \leq \ldots \leq i_{k} \leq n}\left(1-\prod_{j=1}^{n}\left(1-\left(v_{i_{j}}\right)^{2}\right)^{\rho_{i_{j}}}\right)\right)^{\left(1 / \mathbb{C}_{n}^{k}\right)}\right)^{(1 / k)}\right)\right\} .}
\end{aligned}
$$

\section{Countersection 4 of [19]}

This section recalls the discussion of DMSM AO for CPLFN and their basic properties proposed by Fahmi et al. [19].

9.1. Weighted DMSM Averaging. Definition 4.1.1 and Theorem 4.1.2 in [19] proposed the weighted DMSM averaging operator using defined operational rules (Definition 15) described as follows.

Definition 28 (see [19]). Let $\mathscr{F}_{\mathrm{cp}_{i}}=\left\{\ell_{v_{i}},\left\langle\left[\mu_{i}^{-}(v)\right.\right.\right.$, $\left.\left.\left.\mu_{i}^{+}(v)\right], \mu_{i}(v)\right\rangle\right\} ;(i \in \mathbb{N}) \in \operatorname{CPLFS}(U)$ and $\left(\rho_{1}, \rho_{2}, \ldots, \rho_{n}\right)^{T}$ is weight vector having $\rho_{\natural} \geq 0$ and $\sum_{\natural=1}^{n} \rho_{\natural}=1$. Then, the CPLFDWMSMA operator is described as follows: 


$$
\operatorname{CPLFDWMSMA}\left(\mathscr{F}_{\mathrm{cp}_{1}}, \mathscr{F}_{\mathrm{cp}_{2}}, \ldots, \mathscr{F}_{\mathrm{cp}_{n}}\right)=\left(\frac{1}{k}\left(\sum_{\theta \in S_{n}}\left(\sum_{i=1}^{n} \rho_{j} \mathscr{F}_{\mathrm{cp}_{\theta(i)}}\right)\right)\right)^{(1 / k)}
$$

where $\theta(i)(i \in \mathbb{N})$ represents any permutation and $S_{n}$ represents the total numbers of elements.
Theorem 10 (see [19]). Let $\mathscr{F}_{c p_{i}}=\left\{\ell_{v_{i}},\left\langle\left[\mu_{i}^{-}(v)\right.\right.\right.$, $\left.\left.\left.\mu_{i}^{+}(v)\right], \mu_{i}(v)\right\rangle\right\} ;(i \in \mathbb{N}) \in C P L F S(U)$. Then, using defined operational laws, the CPLFDWMSMA operator is obtained as

$$
\begin{aligned}
& \operatorname{CPLFDWMSMA}\left(\mathscr{F}_{\mathrm{cp}_{1}}, \mathscr{F}_{\mathrm{cp}_{2}}, \ldots, \mathscr{F}_{\mathrm{cp}_{n}}\right) \\
& =\left\{\begin{array}{l}
\ell \\
(1 / k)\left(\left(1 / \mathbb{C}_{n}^{k}\right)\left(\sum_{1 \leq i_{1} \leq i_{2} \leq \cdots \leq i_{k} \leq n} \prod_{i=1}^{k} h_{\theta(i)}^{\rho_{i}}\right)\right)^{\left(1 / \mathbb{C}_{n}^{k}\right)}
\end{array}\right. \\
& \cdot\left[\sqrt{1-\left(1-\left(\prod_{1 \leq i_{1} \leq i_{2} \leq \cdots \leq i_{k} \leq n}\left(1-\prod_{i=1}^{n}\left(1-\left(\mu_{i}^{-}\right)^{2}\right)^{\rho_{i}}\right)\right)^{\left(1 / \mathbb{C}_{n}^{k}\right)}\right)^{(1 / k)}}\right. \\
& \left.\sqrt{1-\left(1-\left(\prod_{1 \leq i_{1} \leq i_{2} \leq \cdots \leq i_{k} \leq n}\left(1-\prod_{i=1}^{n}\left(1-\left(\mu_{i}^{+}\right)^{2}\right)^{\rho_{i}}\right)\right)^{\left(1 / \mathbb{C}_{n}^{k}\right)}\right)^{(1 / k)}}\right] \\
& \left.\sqrt{\left(1-\prod_{1 \leq i_{1} \leq i_{2} \leq \ldots \leq i_{k} \leq n}\left(1-\prod_{i=1}^{n}\left(1-\left(1-\mu_{i}^{2}\right)\right)^{\rho_{i}}\right)^{\left(1 / \mathbb{C}_{n}^{k}\right)}\right)^{(1 / k)}}\right\} \text {. }
\end{aligned}
$$

Definition 4.2.1 and Theorem 4.2.2 in [19] proposed the ordered weighted DMSM averaging operator using defined operational rules (Definition 15) described as follows.
Definition 29 (see [19]). Let $\mathscr{F}_{\mathrm{cp}_{i}}=\left\{\ell_{v_{i}},\left\langle\left[\mu_{i}^{-}(v), \mu_{i}^{+}(v)\right]\right.\right.$, $\left.\left.\mu_{i}(v)\right\rangle\right\} ;(i \in \mathbb{N}) \in \operatorname{CPLFS}(U)$ and $\left(\rho_{1}, \rho_{2}, \ldots, \rho_{n}\right)^{T}$ is weight vector having $\rho_{\natural} \geq 0$ and $\sum_{\natural=1}^{n} \rho_{\natural}=1$. Then, the CPLFDOWMSMA operator is described as follows:

$$
\operatorname{CPLFDOWMSMA}\left(\mathscr{F}_{\mathrm{cp}_{1}}, \mathscr{F}_{\mathrm{cp}_{2}}, \ldots, \mathscr{F}_{\mathrm{cp}_{n}}\right)=\left(\frac{1}{k}\left(\sum_{\theta \in S_{n}}\left(\sum_{i=1}^{n} \rho_{j} \mathscr{F}_{\mathrm{cp}_{\theta(i)}}\right)\right)\right)^{(1 / k)}
$$

where $\theta(i)(i \in \mathbb{N})$ represents any permutation and $S_{n}$ represents the total numbers of elements.
Theorem 11 (see [19]). Let $\mathscr{F}_{c p_{i}}=\left\{\ell_{v_{i}},\left\langle\left[\mu_{i}^{-}(v), \mu_{i}^{+}(v)\right]\right.\right.$, $\left.\left.\mu_{i}(v)\right\rangle\right\} ;(i \in \mathbb{N}) \in C P L F S(U)$. Then, using defined operational laws, the CPLFDOWMSMA operator is obtained as

$$
\begin{aligned}
& \operatorname{CPLFDOWMSMA}\left(\mathscr{F}_{\mathrm{cp}_{1}}, \mathscr{F}_{\mathrm{cp}_{2}}, \ldots, \mathscr{F}_{\mathrm{cp}_{n}}\right)
\end{aligned}
$$

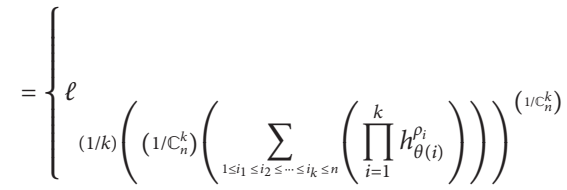

$$
\begin{aligned}
& \cdot\left(\left[\sqrt{1-\left(1-\left(\prod_{1 \leq i_{1} \leq i_{2} \leq \ldots \leq i_{k} \leq n}\left(1-\prod_{i=1}^{n}\left(1-\left(\mu_{i}^{-}\right)^{2}\right)^{\rho_{i}}\right)\right)^{\left(1 / \mathbb{C}_{n}^{k}\right)}\right)^{(1 / k)}}, \sqrt{1-\left(1-\left(\prod_{1 \leq i_{1} \leq i_{2} \leq \ldots \leq i_{k} \leq n}\left(1-\prod_{i=1}^{n}\left(1-\left(\mu_{i}^{+}\right)^{2}\right)^{\rho_{i}}\right)\right)^{\frac{1}{\mathbb{C}_{n}^{k}}}\right)^{(1 / k)}}\right.\right. \\
& \left.\left.\sqrt{\left(1-\prod_{1 \leq i_{1} \leq i_{2} \leq \cdots \leq i_{k} \leq n}\left(1-\prod_{i=1}^{n}\left(1-\left(1-\mu_{i}^{2}\right)\right)^{\rho_{i}}\right)^{\left(1 / \mathbb{C}_{n}^{k}\right)}\right)^{(1 / k)}}\right)\right\} \text {. }
\end{aligned}
$$


Definition 4.3.1 and Theorem 4.3.2 in [19] proposed the hybrid weighted DMSM averaging operator using defined operational rules (Definition 15) described as follows.
Definition 30 (see [19]). Let $\mathscr{F}_{\mathrm{cp}_{i}}=\left\{\ell_{v_{i}},\left\langle\left[\mu_{i}^{-}(v), \mu_{i}^{+}(v)\right], \mu_{i}\right.\right.$ $(v)\rangle\} ;(i \in \mathbb{N}) \in \operatorname{CPLFS}(U)$ and $\left(\rho_{1}, \rho_{2}, \ldots, \rho_{n}\right)^{T}$ is associated weight vector having $\rho_{\natural} \geq 0$ and $\sum_{\natural=1}^{n} \rho_{\natural}=1$. Then, the CPLFDHWMSMA operator is described as follows:

$$
\operatorname{CPLFDHWMSMA}\left(\mathscr{F}_{\mathrm{cp}_{1}}, \mathscr{F}_{\mathrm{cp}_{2}}, \ldots, \mathscr{F}_{\mathrm{cp}_{n}}\right)=\left(\frac{1}{k}\left(\sum_{\theta \in S_{n}}\left(\sum_{i=1}^{n} \rho_{j} \mathscr{F}_{c p_{\theta(i)}}\right)\right)^{(1 / k)}\right.
$$

where $\theta(i)(i \in \mathbb{N})$ represented the any permutation and $S_{n}$ represented the total numbers of elements.
Theorem 12 (see [19]). Let $\mathscr{F}_{c p_{i}}=\left\{\ell_{v_{i}},\left\langle\left[\mu_{i}^{-}(v), \mu_{i}^{+}(v)\right]\right.\right.$, $\left.\left.\mu_{i}(v)\right\rangle\right\} ;(i \in \mathbb{N}) \in C P L F S(U)$. Then, using defined operational laws, the CPLFDHWMSMA operator is obtained as

$$
\begin{aligned}
& \operatorname{CPLFDHWMSMA}\left(\mathscr{F}_{\mathrm{cp}_{1}}, \mathscr{F}_{\mathrm{cp}_{2}}, \ldots, \mathscr{F}_{\mathrm{cp}_{n}}\right) \\
& =\left\{\begin{array}{l}
\ell \\
(1 / k)\left(\left(1 / \mathbb{C}_{n}^{k}\right)\left(\sum_{1 \leq i_{1} \leq i_{2} \leq \cdots \leq i_{k} \leq n} \prod_{i=1}^{k} h_{\theta(i)}^{\rho_{i}}\right)\right)^{\left(1 / \mathbb{C}_{n}^{k}\right)}
\end{array}\right. \\
& \cdot\left(\left[\sqrt{1-\left(1-\left(\prod_{1 \leq i_{1} \leq i_{2} \leq \cdots \leq i_{k} \leq n}\left(1-\prod_{i=1}^{n}\left(1-\left(\mu_{i}^{-}\right)^{2}\right)^{\rho_{i}}\right)\right)^{\left(1 / \mathbb{C}_{n}^{k}\right)}\right)^{(1 / k)}}\right.\right. \\
& \text {. } \sqrt{1-\left(1-\left(\prod_{1 \leq i_{1} \leq i_{2} \leq \cdots \leq i_{k} \leq n}\left(1-\prod_{i=1}^{n}\left(1-\left(\mu_{i}^{+}\right)^{2}\right)^{\rho_{i}}\right)\right)^{\left(1 / \mathbb{C}_{n}^{k}\right)}\right)^{(1 / k)}} \\
& \text {. } \left.\left.\sqrt{\left(1-\prod_{1 \leq i_{1} \leq i_{2} \leq \cdots \leq i_{k} \leq n}\left(1-\prod_{i=1}^{n}\left(1-\left(1-\$ \mu_{i}^{2} \$\right)\right)^{\rho_{i}}\right)^{\left(1 / \mathbb{C}_{n}^{k}\right)}\right)^{(1 / k)}}\right)\right\} \text {. }
\end{aligned}
$$

9.2. Weighted DMSM Geometric. Definition 4.4.1 and Theorem 4.4.2 in [19] proposed the weighted DMSM geometric operator using defined operational rules (Definition 15) described as follows.
Definition 31 (see [19]). Let $\mathscr{F}_{\mathrm{cp}_{i}}=\left\{\ell_{v_{i}},\left\langle\left[\mu_{i}^{-}(v), \mu_{i}^{+}(v)\right]\right.\right.$, $\left.\left.\mu_{i}(v)\right\rangle\right\} ;(i \in \mathbb{N}) \in \operatorname{CPLFS}(U)$ and $\left(\rho_{1}, \rho_{2}, \ldots, \rho_{n}\right)^{T}$ is weight vector having $\rho_{\natural} \geq 0$ and $\sum_{\natural=1}^{n} \rho_{\natural}=1$. Then, the CPLFDWMSMG operator is described as follows:

$$
\operatorname{CPLFDWMSMG}\left(\mathscr{F}_{\mathrm{cp}_{1}}, \mathscr{F}_{\mathrm{cp}_{2}}, \ldots, \mathscr{F}_{\mathrm{cp}_{n}}\right)=\left(\frac{1}{k}\left(\prod_{\theta \in S_{n}}\left(\prod_{i=1}^{n} \mathscr{F}_{{ }_{\mathrm{c} \mathrm{p}_{\theta(i)}} \rho_{i}}\right)\right)\right)^{(1 / k)},
$$

where $\theta(i)(i \in \mathbb{N})$ represents any permutation and $S_{n}$ represents total numbers of elements. 
Theorem 13 (see [19]). Let $\mathscr{F}_{c p_{i}}=\left\{\ell_{v_{i}},\left\langle\left[\mu_{i}^{-}(v), \mu_{i}^{+}(v)\right]\right.\right.$, $\left.\left.\mu_{i}(v)\right\rangle\right\} ;(i \in \mathbb{N}) \in \operatorname{CPLFS}(U)$. Then, using defined operational laws, the CPLFDWMSMG operator is obtained as

$$
\begin{aligned}
& \operatorname{CPLFDWMSMG}\left(\mathscr{F}_{\mathrm{cp}_{1}}, \mathscr{F}_{\mathrm{cp}_{2}}, \ldots, \mathscr{F}_{\mathrm{cp}_{n}}\right) \\
& =\left\{\begin{array}{l}
\ell \\
(1 / k)\left(\left(1 / \mathbb{C}_{n}^{k}\right)\left(\sum_{1 \leq i_{1} \leq i_{2} \leq \ldots \leq i_{k} \leq n} \prod_{i=1}^{k} h_{\theta(i)}^{\rho_{i}}\right)\right)^{\left(1 / c_{n}^{k}\right)}
\end{array}\right. \\
& \cdot\left[\sqrt{\left(\prod_{1 \leq i_{1} \leq i_{2} \leq \cdots \leq i_{k} \leq n}\left(\prod_{i=1}^{n}\left(\left(\mu_{i}^{-}\right)^{2}\right)^{\rho_{i}}\right)^{\left(1 / \mathbb{C}_{n}^{k}\right)}\right)^{(1 / k)}}, \sqrt{\left(\prod_{1 \leq i_{1} \leq i_{2} \leq \cdots \leq i_{k} \leq n}\left(\prod_{i=1}^{n}\left(\left(\mu_{i}^{+}\right)^{2}\right)^{\rho_{i}}\right)^{\left(1 / \mathbb{C}_{n}^{k}\right)}\right)^{(1 / k)}}\right], \\
& \left.\left.\cdot\left(\sqrt{\prod_{1 \leq i_{1} \leq i_{2} \leq \cdots \leq i_{k} \leq n}\left(1-\prod_{i=1}^{n}\left(1-\left(\mu_{i}^{2}\right)\right)^{\rho_{i}}\right)^{\left(1 / \mathbb{C}_{n}^{k}\right)}}\right)^{(1 / k)}\right)\right\} .
\end{aligned}
$$

Definition 4.5.1 and Theorem 4.5.2 in [19] proposed the ordered weighted DMSM geometric operator using defined operational rules (Definition 15) is described as
Definition 32 (see [19]). Let $\mathscr{F}_{\mathrm{cp}_{i}}=\left\{\ell_{v_{i}},\left\langle\left[\mu_{i}^{-}(v), \mu_{i}^{+}(v)\right]\right.\right.$, $\left.\left.\mu_{i}(v)\right\rangle\right\} ;(i \in \mathbb{N}) \in \operatorname{CPLFS}(U)$ and $\left(\rho_{1}, \rho_{2}, \ldots, \rho_{n}\right)^{T}$ is weight vector having $\rho_{\natural} \geq 0$ and $\sum_{\natural=1}^{n} \rho_{\natural}=1$. Then, the CPLFDOWMSMG operator is described as follows:

$$
\operatorname{CPLFDOWMSMG}\left(\mathscr{F}_{\mathrm{cp}_{1}}, \mathscr{F}_{\mathrm{cp}_{2}}, \ldots, \mathscr{F}_{\mathrm{cp}_{n}}\right)=\left(\frac{1}{k}\left(\prod_{\theta \in S_{n}}\left(\prod_{i=1}^{n} \mathscr{F}_{\mathrm{cp}_{\theta(i)}}^{\rho_{i}}\right)\right)^{(1 / k)}\right.
$$

where $\theta(i)(i \in \mathbb{N})$ represents any permutation and $S_{n}$ represents the total numbers of elements.
Theorem 14 (see [19]). Let $\mathscr{F}_{c p_{i}}=\left\{\ell_{v_{i}},\left\langle\left[\mu_{i}^{-}(v), \mu_{i}^{+}(v)\right]\right.\right.$, $\left.\left.\mu_{i}(v)\right\rangle\right\} ;(i \in \mathbb{N}) \in \operatorname{CPLFS}(U)$. Then, using defined operational laws, the CPLFDOWMSMG operator is obtained as

$$
\begin{aligned}
& \operatorname{CPLFDOWMSMG}\left(\mathscr{F}_{\mathrm{cp}_{1}}, \mathscr{F}_{\mathrm{cp}_{2}}, \ldots, \mathscr{F}_{\mathrm{cp}_{n}}\right)
\end{aligned}
$$

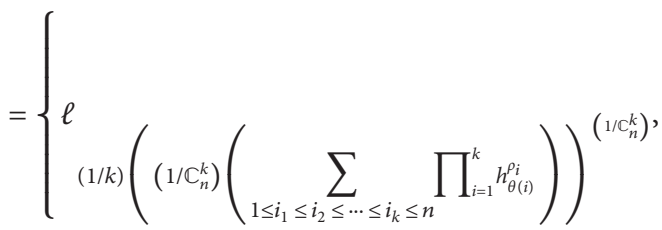

$$
\begin{aligned}
& \cdot\left(\left[\sqrt{\left(\prod_{1 \leq i_{1} \leq i_{2} \leq \cdots \leq i_{k} \leq n}\left(\prod_{i=1}^{n}\left(\left(\mu_{i}^{-}\right)^{2}\right)^{\rho_{i}}\right)^{\left(1 / \mathbb{C}_{n}^{k}\right)}\right)^{(1 / k)}}, \sqrt{\left(\prod_{1 \leq i_{1} \leq i_{2} \leq \cdots \leq i_{k} \leq n}\left(\prod_{i=1}^{n}\left(\left(\mu_{i}^{+}\right)^{2}\right)^{\rho_{i}}\right)^{\left(1 / \mathbb{C}_{n}^{k}\right)}\right)^{(1 / k)}}\right],\right. \\
& \left.\left.\cdot\left(\sqrt{\prod_{1 \leq i_{1} \leq i_{2} \leq \cdots \leq i_{k} \leq n}\left(1-\prod_{i=1}^{n}\left(1-\left(\mu_{i}^{2}\right)\right)^{\rho_{i}}\right)^{\left(1 / \mathbb{C}_{n}^{k}\right)}}\right)^{(1 / k)}\right)\right\} \text {. }
\end{aligned}
$$


Definition 4.6.1 and Theorem 4.6.2 in [19] proposed the hybrid-weighted DMSM geometric operator using defined operational rules (Definition 15) described as follows.
Definition 33 (see [19]). Let $\mathscr{F}_{\mathrm{cp}_{i}}=\left\{\ell_{v_{i}},\left\langle\left[\mu_{i}^{-}(v), \mu_{i}^{+}(v)\right]\right.\right.$, $\left.\left.\mu_{i}(v)\right\rangle\right\} ;(i \in \mathbb{N}) \in \operatorname{CPLFS}(U)$ and $\left(\rho_{1}, \rho_{2}, \ldots, \rho_{n}\right)^{T}$ is weight vector having $\rho_{\natural} \geq 0$ and $\sum_{\natural=1}^{n} \rho_{\natural}=1$. Then, the CPLFDHWMSMG operator is described as follows:

$$
\operatorname{CPLFDHWMSMG}\left(\mathscr{F}_{\mathrm{cp}_{1}}, \mathscr{F}_{\mathrm{cp}_{2}}, \ldots, \mathscr{F}_{\mathrm{cp}_{n}}\right)=\left(\frac{1}{k}\left(\prod_{\theta \in S_{n}}\left(\prod_{i=1}^{n} \mathscr{F}_{\mathrm{cp}_{\theta(i)}}^{\rho_{i}}\right)\right)^{(1 / k)}\right.
$$

where $\theta(i)(i \in \mathbb{N})$ represents any permutation and $S_{n}$ represents the total numbers of elements.

$$
\begin{aligned}
& \operatorname{CPLFDHWMSMG}\left(\mathscr{F}_{\mathrm{cp}_{1}}, \mathscr{F}_{\mathrm{cp}_{2}}, \ldots, \mathscr{F}_{\mathrm{cp}_{n}}\right) \\
& =\left\{\begin{array}{l}
\ell \\
(1 / k)\left(\left(1 / \mathbb{C}_{n}^{k}\right)\left(\sum_{1 \leq i_{1} \leq i_{2} \leq \cdots \leq i_{k} \leq n} \prod_{i=1}^{k} h_{\theta(i)}^{\rho_{i}}\right)\right)^{\left(1 / \mathbb{C}_{n}^{k}\right)}
\end{array}\right. \\
& \cdot\left(\left[\sqrt{\left(\prod_{1 \leq i_{1} \leq i_{2} \leq \cdots \leq i_{k} \leq n}\left(\prod_{i=1}^{n}\left(\left(\mu_{i}^{-}\right)^{2}\right)^{\rho_{i}}\right)^{\left(1 / \mathbb{C}_{n}^{k}\right)}\right)^{(1 / k)}}, \sqrt{\left(\prod_{1 \leq i_{1} \leq i_{2} \leq \cdots \leq i_{k} \leq n}\left(\prod_{i=1}^{n}\left(\left(\mu_{i}^{+}\right)^{2}\right)^{\rho_{i}}\right)^{\left(1 / \mathbb{C}_{n}^{k}\right)}\right)^{(1 / k)}}\right],\right. \\
& \left.\left.\cdot\left(\sqrt{\prod_{1 \leq i_{1} \leq i_{2} \leq \cdots \leq i_{k} \leq n}\left(1-\prod_{i=1}^{n}\left(1-\left(\mu_{i}^{2}\right)\right)^{\rho_{i}}\right)^{\left(1 / \mathbb{C}_{n}^{k}\right)}}\right)^{(1 / k)}\right)\right\} .
\end{aligned}
$$

Theorem 15 (see [19]). Let $\mathscr{F}_{c p_{i}}=\left\{\ell_{v_{i}},\left\langle\left[\mu_{i}^{-}(v), \mu_{i}^{+}(v)\right]\right.\right.$, $\left.\left.\mu_{i}(v)\right\rangle\right\} ;(i \in \mathbb{N}) \in \operatorname{CPLFS}(U)$. Then, using defined operational laws, the CPLFDHWMSMG operator is obtained as

\section{Updated Dual MSM Operators}

In this section, valid Definition 16 of LCPFS and operational laws (Definition 17) are utilized to establish the updated linguistic dual Maclaurin symmetric mean AO to aggregate the uncertain data in the form of linguistic cubic Pythagorean fuzzy environment.

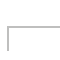

Definition 34. Let $\mathscr{F}_{\mathrm{cp}_{i}}=\left\{\ell_{v_{i}},\left(\left(\left[\mu_{i}^{-}(v), \mu_{i}^{+}(v)\right],\left[v_{i}^{-}\right.\right.\right.\right.$ $\left.\left.\left.\left.(v), v_{i}^{+}(v)\right]\right), \quad\left(\mu_{i}(v), v_{i}(v)\right)\right)\right\} ;(i \in \mathbb{N}) \in \operatorname{CPLFS}(U) \quad$ and $\left(\rho_{1}, \rho_{2}, \ldots, \rho_{n}\right)^{T}$ is weight vector having $\rho_{\natural} \geq 0$ and $\sum_{\natural=1}^{n} \rho_{\natural}=1$. Then, the CPLFWDMSM operator is described as follows:

$$
\operatorname{CPLFWDMSM}\left(\mathscr{F}_{\mathrm{cp}_{1}}, \mathscr{F}_{\mathrm{cp}_{2}}, \ldots, \mathscr{F}_{\mathrm{cp}_{n}}\right)=\frac{1}{k}\left(\prod_{1 \leq i_{1} \leq i_{2} \leq \cdots \leq i_{k} \leq n}\left(\sum_{j=1}^{k}\left(\rho_{i_{j}} \otimes \mathscr{F}_{\mathrm{cp}_{i_{j}}}\right)\right)^{\left(1 / \mathbb{C}_{n}^{k}\right)}\right),
$$

where $\mathbb{C}_{n}^{k}$ is the binomial coefficient and $\left(i_{1}, i_{2}, \ldots, i_{k}\right)$ traversal of all the k-tuple combination of $(1,2, \ldots, n)$.
Theorem 16. Let $\mathscr{F}_{c p_{i}}=\left\{\ell_{v_{i}},\left(\left(\left[\mu_{i}^{-}(v), \mu_{i}^{+}(v)\right],\left[v_{i}^{-}\right.\right.\right.\right.$ $\left.\left.\left.\left.(v), v_{i}^{+}(v)\right]\right), \quad\left(\mu_{i}(v), v_{i}(v)\right)\right)\right\} ;(i \in \mathbb{N})^{+} \in \operatorname{CPLFS}(U)$. Then, 
using defined operational laws 17, the CPLFWDMSM operator

is obtained as

$$
\begin{aligned}
& \operatorname{CPLFWDMSM}\left(\mathscr{F}_{\mathrm{cp}_{1}}, \mathscr{F}_{\mathrm{cp}_{2}}, \ldots, \mathscr{F}_{\mathrm{cp}_{n}}\right)=\frac{1}{k}\left(\prod_{1 \leq i_{1} \leq i_{2} \leq \cdots \leq i_{k} \leq n}\left(\sum_{j=1}^{k}\left(\rho_{i_{j}} \otimes \mathscr{F}_{\mathrm{cp}_{i_{j}}}\right)\right)^{\left(1 / \mathbb{C}_{n}^{k}\right)}\right)
\end{aligned}
$$

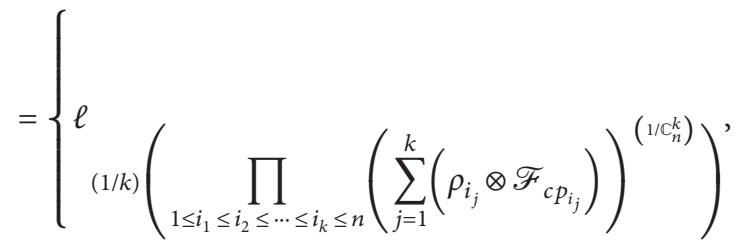

$$
\begin{aligned}
& \cdot\left(\left[\sqrt{1-\left(1-\left(\prod_{1 \leq i_{1} \leq i_{2} \leq \cdots \leq i_{k} \leq n}\left(1-\prod_{j=1}^{n}\left(1-\left(\mu_{i_{j}}^{-}\right)^{2}\right)^{\rho_{i_{j}}}\right)\right)^{\left(1 / \mathbb{C}_{n}^{k}\right)}\right)^{(1 / k)}},\right.\right. \\
& \sqrt{1-\left(1-\left(\prod_{1 \leq i_{1} \leq i_{2} \leq \cdots \leq i_{k} \leq n}\left(1-\prod_{j=1}^{n}\left(1-\left(\mu_{i_{j}}^{+}\right)^{2}\right)^{\rho_{i_{j}}}\right)\right)^{\left(1 / \mathbb{C}_{n}^{k}\right)}\right)^{(1 / k)}} \\
& {\left[\left(\sqrt{1-\prod_{1 \leq i_{1} \leq i_{2} \leq \cdots \leq i_{k} \leq n}\left(1-\left(\prod_{j=1}^{n}\left(v_{i_{j}}^{-}\right)^{\rho_{i_{j}}}\right)^{2}\right)^{\left(1 / \mathbb{C}_{n}^{k}\right)}}\right)^{(1 / k)},\right.} \\
& \left.\left(\sqrt{1-\prod_{1 \leq i_{1} \leq i_{2} \leq \cdots \leq i_{k} \leq n}\left(1-\left(\prod_{j=1}^{n}\left(v_{i_{j}}^{+}\right)^{\rho_{i_{j}}}\right)^{2}\right)^{\left(1 / \mathbb{C}_{n}^{k}\right)}}\right)^{(1 / k)}\right], \\
& \left(\sqrt{1-\left(1-\left(\prod_{1 \leq i_{1} \leq i_{2} \leq \cdots \leq i_{k} \leq n}\left(1-\prod_{j=1}^{n}\left(1-\left(\mu_{i_{j}}\right)^{2}\right)^{\rho_{i_{j}}}\right)\right)^{\left(1 / \mathbb{C}_{n}^{k}\right)}\right)^{(1 / k)}},\right. \\
& \left.\left.\left.\left(\sqrt{1-\prod_{1 \leq i_{1} \leq i_{2} \leq \ldots \% \leq i_{k} \leq n}\left(1-\left(\prod_{j=1}^{n}\left(v_{i_{j}}\right)^{\rho_{i_{j}}}\right)^{2}\right)^{\left(1 / \mathbb{C}_{n}^{k}\right)}}\right)^{(1 / k)}\right)\right)\right\} .
\end{aligned}
$$

\section{Decision-Making Model Based on Updated MSM Operators}

In this section, we propose a framework for solving multiattribute decision-making problems (DMPs) under cubic
Pythagorean linguistic fuzzy (CPLF) information. Consider a MADM with a set of $m$ alternatives $\left\{\beth_{1}, \beth_{2}, \ldots, \beth_{h}\right\}$, and let $\left\{y_{1}, y_{2}, \ldots, y_{h}\right\}$ be a set of attributes with weight vector $p=\left(p_{1}, p_{2}, \ldots, p_{h}\right)$, where $p_{t} \in[0,1]$ and $\sum_{t=1}^{h} p_{t}=1$. To assess the performance of $k$ th alternative $\lambda_{k}$ under the $t$ th 
attribute $y_{t}$, let $\left\{\stackrel{\circ}{D}_{1}, \stackrel{\circ}{D}_{2}, \ldots, \stackrel{\circ}{D}_{\mathcal{J}}\right\}$ be a set of decision makers and $\widehat{w}=\left(\widehat{w}_{1}, \widehat{w}_{2}, \ldots, \widehat{w}_{\mathcal{F}}\right)$ be the weighted vector of decision makers with $\widehat{w}_{s} \in[0,1]$ and $\sum_{s=1}^{\mathscr{J}} \widehat{w}_{s}=1$. The CPLF decision matrix can be written as

$$
\left[\begin{array}{ccccc}
b_{11} & b_{12} & b_{13} & \cdots & b_{1 h} \\
b_{21} & b_{22} & b_{23} & \cdots & b_{2 h} \\
b_{31} & b_{32} & b_{33} & \cdots & b_{3 h} \\
\vdots & \vdots & \vdots & \ddots & \vdots \\
b_{\natural 1} & b_{\natural 2} & b_{\natural 3} & \cdots & b_{\natural h}
\end{array}\right],
$$

where $b_{i j}=\left\{\ell_{v_{i j}},\left(\left(\left[\mu_{i j}^{-}(v), \mu_{i j}^{+}(v)\right],\left[v_{i j}^{-}(v), v_{i j}^{+}(v)\right]\right),\left(\mu_{i j}(v)\right.\right.\right.$, $\left.\left.\left.v_{i j}(v)\right)\right)\right\}$ and $\left[\mu^{-}(v), \mu^{+}(v)\right],\left[v^{-}(v), v^{+}(v)\right] \subseteq[0,1]$ and $\mu(v), v(v) \in[0,1]$ represented the positive membership grade in the form of interval-valued Pythagorean fuzzy set and negative membership grade in the form of Pythagorean fuzzy set. Key steps of the developed multiattribute decisionmaking (MADM) problem are described as follows.

Step 1: construct the CPLF decision matrix based on the experts' evaluations:

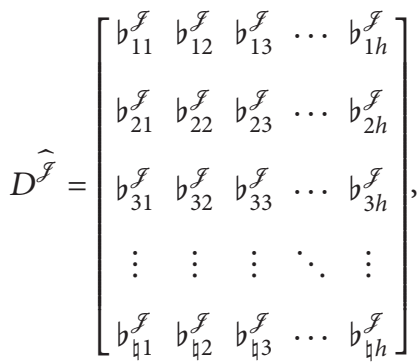

where $\mathscr{J}$ represents the number of expert.

Step 2: exploit the established aggregation operators to achieve the CPLFNs $\mathscr{F}_{t}(t=1,2, \ldots, q)$ for the alternatives $I_{k}$, that is, the established operators to obtain the collective overall preference values of $\mathscr{F}_{t}(t=1,2$, $\ldots, q)$ for the alternatives $z_{k}$, where $p=\left(p_{1}, p_{2}, \ldots\right.$, $\left.p_{h}\right)$ is the weight vector of the attributes.

Step 3: after that, we compute the scores of all the overall values $\mathscr{F}_{t}(t=1,2, \ldots, q)$ for the alternatives $\beth_{k}$. Step 4: according to Definition 18, rank the alternatives $\beth_{k}(k=1,2, \ldots, \emptyset)$ and select the best one having the greater value.

\section{Numerical Application}

The company of intranet is usually attacked by malicious intrusions. To enhance the security of the intranet, the company plans to purchase the firewall production and put it between the intranet and extranet for blocking illegal access. Basically, there are four types of firewall productions given to be considered, whose detailed is as follows: $C=\left\{C_{1}, C_{2}, C_{3}, C_{4}\right\}$. If the firewall production is chosen, the company pays attention to the factors, which are detailed as follows: $G_{1} \longrightarrow$ the promotion, $G_{2} \longrightarrow$ configuration simplicity, $G_{3} \longrightarrow$ security level, and $G_{4} \longrightarrow$ maintenance server level, whose weight vector is $(0.2,0.1,0.3,0.4)^{T}$. To examine the firewall production with respect to their factor, we consider the cubic Pythagorean linguistic fuzzy matrix, the decision matrix is given in the form of Table 1:

Step 1: the evaluation result of the expert is listed in Table 1:

Step 2: based on the proposed MSM operators, the collective CPLF information of each alternative is obtained as follows in Tables 2-4:

$b_{11} \otimes b_{12}=\left\{\ell_{8},([0.15,0.48],[0.48,0.75])(0.21,0.44)\right\}$,

$b_{11} \otimes b_{13}=\left\{\ell_{2},([0.10,0.32],[0.48,0.68])(0.14,0.22)\right\}$,

$b_{11} \otimes b_{14}=\left\{\ell_{10},([0.20,0.40],[0.64,0.75])(0.42,0.52)\right\}$,

$b_{12} \otimes b_{13}=\left\{\ell_{4},([0.06,0.24],[0.54,0.82])(0.06,0.41)\right\}$,

$b_{12} \otimes b_{14}=\left\{\ell_{20},([0.12,0.30],[0.68,0.86])(0.18,0.60)\right\}$,

$b_{13} \otimes b_{14}=\left\{\ell_{5},([0.08,0.20],[0.68,0.82])(0.12,0.50)\right\}$.

Case 1: from Table 1, we have $b_{11}=\left\{\ell_{2},([0.5,0.8]\right.$, $[0.3,0.4])(0.7,0.2)\}, \quad b_{12}=\left\{\ell_{4},([0.3,0.6],[0.4,0.7])\right.$ $(0.3,0.4)\}, \quad b_{13}=\left\{\ell_{1},([0.2,0.4],[0.4,0.6])(0.2,0.1)\right\}$, and $b_{14}=\left\{\ell_{5},([0.4,0.5],[0.6,0.7])(0.6,0.5)\right\} \in$ CPLFS $(U)$. Here, we use CPLFMSM operator to aggregate the expert evaluation in the form of CPLFSs. Without loss of generality, we take $k=2$; then,

Using Theorem 8 , we obtain

$$
\begin{aligned}
& \operatorname{CPLFMSM}^{(2)}\left(b_{11}, b_{12}, b_{13}, b_{14}\right) \\
& =\left(\frac{\sum_{1 \leq i_{1} \leq i_{2} \leq 4}\left(b_{i_{1}} \otimes b_{i_{2}}\right)}{\mathbb{C}_{4}^{2}}\right)^{(1 / 2)} \\
& =\left\{\ell_{1.52},([0.242,0.438],[0.352,0.530])(0.337,0.246)\right\} .
\end{aligned}
$$

Hence, we obtained similarly. 


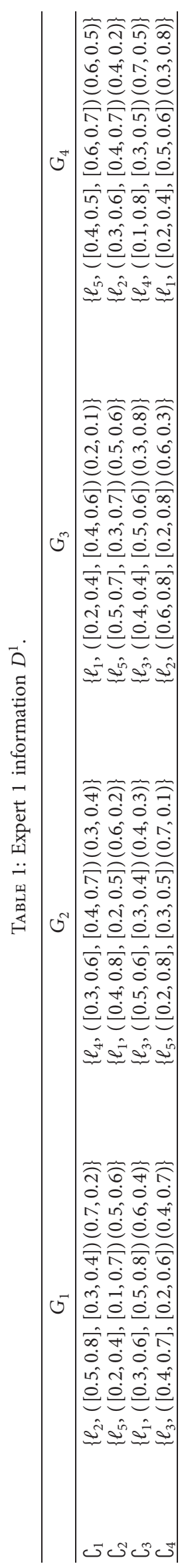


TABLE 2: CPLFMSM ${ }^{(2)}$ operator.

\begin{tabular}{ll}
\hline$C_{1}$ & $\left\{\ell_{1.520},([0.242,0.438],[0.352,0.530])(0.337,0.246)\right\}$ \\
\hline$C_{2}$ & $\left\{\ell_{1.568},([0.242,0.493],[0.200,0.578])(0.362,0.339)\right\}$ \\
$C_{3}$ & $\left\{\ell_{1.482},([0.231,0.464],[0.329,0.506])(0.371,0.432)\right\}$ \\
$C_{4}$ & $\left\{\ell_{1.468},([0.246,0.555],[0.239,0.553])(0.372,0.431)\right\}$ \\
\hline
\end{tabular}

TABLE 3: CPLFWMSM(2) operator.

\begin{tabular}{ll}
\hline$C_{1}$ & $\left\{\ell_{1.111},([0.621,0.817],[0.017,0.041])(0.762,0.027)\right\}$ \\
\hline$C_{2}$ & $\left\{\ell_{1.118},([0.655,0.812],[0.005,0.057])(0.744,0.014)\right\}$ \\
$C_{3}$ & $\left\{\ell_{1.105},([0.658,0.787],[0.020,0.046])(0.725,0.007)\right\}$ \\
$C_{4}$ & $\left\{\ell_{1.061},([0.665,0.841],[0.008,0.051])(0.735,0.016)\right\}$ \\
\hline
\end{tabular}

$$
\begin{aligned}
& \left(b_{11}\right)^{0.2} \otimes\left(b_{12}\right)^{0.1}=\left\{\ell_{1.319},([0.77,0.90],[0.01,0.04])(0.82,0.01)\right\} \\
& \left(b_{11}\right)^{0.2} \otimes\left(b_{13}\right)^{0.3}=\left\{\ell_{1.148},([0.53,0.73],[0.03,0.06])(0.57,0.004)\right\} \\
& \left(b_{11}\right)^{0.2} \otimes\left(b_{14}\right)^{0.4}=\left\{\ell_{2.186},([0.60,0.72],[0.05,0.08])(0.75,0.02)\right\} \\
& \left(b_{12}\right)^{0.1} \otimes\left(b_{13}\right)^{0.3}=\left\{\ell_{1.148},([0.54,0.72],[0.02,0.09])(0.54,0.007)\right\} \\
& \left(b_{12}\right)^{0.1} \otimes\left(b_{14}\right)^{0.4}=\left\{\ell_{2.186},([0.61,0.72],[0.05,0.12])(0.72,0.04)\right\}, \\
& \left(b_{13}\right)^{0.3} \otimes\left(b_{14}\right)^{0.4}=\left\{\ell_{1.903},([0.42,0.57],[0.09,0.17])(0.50,0.01)\right\} . \\
& \operatorname{CPLFWMSM}^{(2)}\left(b_{11}, b_{12}, b_{13}, b_{14}\right)=\left(\frac{\sum_{1 \leq i_{1} \leq i_{2} \leq 4}\left(\left(b_{i_{1}}\right)^{\rho_{i_{1}}} \otimes\left(b_{i_{2}}\right)^{\rho_{i_{2}}}\right)}{\mathbb{C}_{4}^{2}}\right)^{(1 / 2)} \\
& =\left\{\ell_{1.105},([0.658,0.787],[0.020,0.046])(0.725,0.007)\right\} \text {. } \\
& \operatorname{CPLFWDMSM}^{(2)}\left(b_{11}, b_{12}, b_{13}, b_{14}\right)=\frac{1}{2}\left(\prod_{1 \leq i_{1} \leq i_{2} \leq 4}\left(\left(\rho_{i_{1}} \otimes \mathscr{F}_{c p_{i_{1}}}\right) \oplus\left(\rho_{i_{2}} \otimes \mathscr{F}_{c p_{i_{2}}}\right)\right)^{\left(1 / \mathbb{C}_{4}^{2}\right)}\right) \\
& =\left\{\ell_{0.657},([0.179,0.302],[0.821,0.883])(0.254,0.738)\right\} \text {. }
\end{aligned}
$$

Case 2: from Table 1, we have $b_{11}=\left\{\ell_{2},([0.5,0.8],[0.3\right.$, $0.4])(0.7,0.2)\}, \quad b_{12}=\left\{\ell_{4},([0.3,0.6],[0.4,0.7])(0.3\right.$, $0.4)\}, \quad b_{13}=\left\{\ell_{1},([0.2,0.4],[0.4,0.6])(0.2,0.1)\right\}$, and $b_{14}=\left\{\ell_{5},([0.4,0.5],[0.6,0.7])(0.6,0.5)\right\} \in \operatorname{CPLFS}(U)$, and $(0.2,0.1,0.3,0.4)^{T}$ is the weight vector. Here, we use CPLFWMSM operator to aggregate the expert evaluation in the form of CPLFSs. Without loss of generality, we take $k=2$; then,

Using Theorem 9, we obtain

Hence, we obtained similarly in Table 3 utilizing Theorem 9,

Case 3: From Table 1, we have $b_{11}=\left\{\ell_{2},([0.5,0.8]\right.$, $[0.3,0.4])(0.7,0.2)\}, \quad b_{12}=\left\{\ell_{4},([0.3,0.6],[0.4,0.7])\right.$ $(0.3,0.4)\}, \quad b_{13}=\left\{\ell_{1},([0.2,0.4],[0.4,0.6])(0.2,0.1)\right\}$, and $\quad b_{14}=\left\{\ell_{5},([0.4,0.5],[0.6,0.7])(0.6,0.5)\right\} \in$
$\operatorname{CPLFS}(U)$, and $(0.2,0.1,0.3,0.4)^{T}$ is the weight vector. Here, we use the CPLFWDMSM operator to aggregate the expert evaluation in the form of CPLFSs. Without loss of generality, we take $k=2$; then, using Theorem 16 , we obtain

Hence, we obtained similarly in Table 4 utilizing Theorem 16,

Step 3: compute the score value of the each collective CPLF information of each alternative as in Table 5.

Step 4: select the optimal alternative according the maximum score value as in Table 6:

From this above computational process, we can conclude that the alternative $\complement_{1}$ is the best among the others, and hence, it is highly recommendable to select for the required task/ plan. 
TABLE 4: CPLFWDMSM ${ }^{(2)}$ operator.

\begin{tabular}{ll}
\hline$C_{1}$ & $\left\{\ell_{0.657},([0.179,0.302],[0.821,0.883])(0.254,0.738)\right\}$ \\
\hline$C_{2}$ & $\left\{\ell_{0.796},([0.179,0.339],[0.721,0.908])(0.248,0.808)\right\}$ \\
$C_{3}$ & $\left\{\ell_{0.667},([0.153,0.331],[0.807,0.878])(0.275,0.858)\right\}$ \\
$C_{4}$ & $\left\{\ell_{0.522},([0.187,0.364],[0.752,0.900])(0.249,0.844)\right\}$ \\
\hline
\end{tabular}

TABLE 5: Score value.

\begin{tabular}{lcccc}
\hline & $\mathcal{S}_{\gamma}\left(\complement_{1}\right)$ & $\mathcal{S}_{\gamma}\left(\complement_{2}\right)$ & $\mathcal{S}_{\gamma}\left(\complement_{3}\right)$ & $\mathcal{S}_{\gamma}\left(\complement_{4}\right)$ \\
\hline CPLFMSM $^{(2)}$ & 0.0908732 & -0.099975 & -0.033468 & -0.074122 \\
CPLFWMSM $^{(2)}$ & 0.655047 & 0.637365 & 0.5915109 & 0.638794 \\
CPLFWDMSM $^{(2)}$ & -0.501965 & -0.590133 & -0.550951 & -0.508489 \\
\hline
\end{tabular}

TABLE 6: Ranking results.

\begin{tabular}{|c|c|c|}
\hline & Score ranking & Best alternative \\
\hline $\begin{array}{l}\text { CPLFMSM }^{(2)} \\
\text { CPLFWMSM }^{(2)} \\
\text { CPLFWDMSM }^{(2)}\end{array}$ & $\begin{array}{l}\delta_{\gamma}\left(\complement_{1}\right)>\delta_{\gamma}\left(\complement_{3}\right)>\delta_{\gamma}\left(\complement_{4}\right)>\delta_{\gamma}\left(\complement_{2}\right) \\
\delta_{\gamma}\left(\complement_{1}\right)>\delta_{\gamma}\left(\complement_{4}\right)>\delta_{\gamma}\left(\complement_{2}\right)>\delta_{\gamma}\left(\complement_{3}\right) \\
\delta_{\gamma}\left(\complement_{1}\right)>\delta_{\gamma}\left(\complement_{4}\right)>\delta_{\gamma}\left(\complement_{3}\right)>\delta_{\gamma}\left(\complement_{2}\right)\end{array}$ & $\begin{array}{l}C_{1} \\
C_{1} \\
C_{1}\end{array}$ \\
\hline
\end{tabular}

\section{Conclusion}

In this note, we discussed that Sections 2.3 and 2.4 in [16] incorrectively define the concept of cubic Pythagorean fuzzy set and their basic operational laws by violating to consider membership and nonmembership function, constructing counterdefinition and countertheorem, and then, we proposed the modified versions of operational laws to tackle the uncertain information in the form of CPFS in decision-making problems. Furthermore, we redefine the concept of cubic Pythagorean linguistic fuzzy set (CPLFS) and their basic operational laws and aim to establish the valid aggregation operators under CPLFS information. In addition, we find that Sections 3 and 4 that consist of list of Maclaurin symmetric mean (MSM) and dual MSM aggregation operators are invalid due to incorrect concept of cubic Pythagorean linguistic fuzzy set, and then, we redefined the list of updated MSM and dual MSM aggregation operators in a correct way. Finally, we proposed the improved algorithm-based numerical application to show the effectiveness and applicability of the valid aggregation operators under cubic Pythagorean linguistic fuzzy settings.

\section{Data Availability}

No data were used to support this study.

\section{Ethical Approval}

This article does not contain any studies with human participants or animals performed by any of the authors.

\section{Conflicts of Interest}

The authors declare that they have no conflicts of interest.

\section{Acknowledgments}

The authors would like to thank the Deanship of Scientific Research (DSR) at Umm Al-Qura University for supporting this work by Grant Code: 19-SCI-1-01-0055.

\section{References}

[1] L. A. Zadeh, "Fuzzy sets," Information and Control, vol. 8, no. 3, pp. 338-353, 1965.

[2] T. Mahmood, "A novel approach towards bipolar soft sets and their applications," Journal of Mathematics, vol. 2020, 2020.

[3] K. Attanassov, "Intuitionistic fuzzy sets," Fuzzy Sets and Systems, vol. 20, pp. 87-96, 1986.

[4] K. T. Atanassov, "Interval valued intuitionistic fuzzy sets," in Intuitionistic Fuzzy Sets, pp. 139-177, Physica, Heidelberg,Germany, 1999.

[5] R. R. Yager, "Pythagorean membership grades in multicriteria decision making," IEEE Transactions on Fuzzy Systems, vol. 22, no. 4, pp. 958-965, 2013.

[6] B. C. Cuong and V. Kreinovich, "Picture fuzzy sets-a new concept for computational intelligence problems," in Proceedings of the 2013 third world congress on information and communication technologies (WICT 2013), pp. 1-6, IEEE, Hanoi, Vietnam, December 2013.

[7] S. Ashraf, T. Mahmood, S. Abdullah, and Q. Khan, "Different approaches to multi-criteria group decision making problems for picture fuzzy environment," Bulletin of the Brazilian Mathematical Society, New Series, vol. 50, no. 2, pp. 373-397, 2019.

[8] S. Ashraf, S. Abdullah, and T. Mahmood, "GRA method based on spherical linguistic fuzzy Choquet integral environment and its application in multi-attribute decision-making problems," Mathematical Sciences, vol. 12, no. 4, pp. 263-275, 2018.

[9] S. Ashraf and S. Abdullah, "Spherical aggregation operators and their application in multiattribute group decision-making," International Journal of Intelligent Systems, vol. 34, no. 3, pp. 493-523, 2019. 
[10] S. Ashraf, S. Abdullah, T. Mahmood, F. Ghani, and T. Mahmood, "Spherical fuzzy sets and their applications in multi-attribute decision making problems," Journal of Intelligent \& Fuzzy Systems, vol. 36, no. 3, pp. 2829-2844, 2019.

[11] L. Wang, H. Garg, and N. Li, "Pythagorean fuzzy interactive Hamacher power aggregation operators for assessment of express service quality with entropy weight," Soft Computing, vol. 25, no. 2, pp. 973-993, 2021.

[12] L. Wang and N. Li, "Pythagorean fuzzy interaction power Bonferroni mean aggregation operators in multiple attribute decision making," International Journal of Intelligent Systems, vol. 35, no. 1, pp. 150-183, 2020.

[13] M. A. Khan, S. Ashraf, S. Abdullah, and F. Ghani, "Applications of probabilistic hesitant fuzzy rough set in decision support system," Soft Computing, vol. 24, no. 22, pp. 16759-16774, 2020.

[14] S. Ashraf, S. Abdullah, and S. Khan, "Fuzzy decision support modeling for internet finance soft power evaluation based on sine trigonometric Pythagorean fuzzy information," Journal of Ambient Intelligence and Humanized Computing, vol. 12, no. 2, pp. 3101-3119, 2021.

[15] B. Batool, M. Ahmad, S. Abdullah, S. Ashraf, and R. Chinram, "Entropy based Pythagorean probabilistic hesitant fuzzy decision making technique and its application for Fog-Haze factor assessment problem," Entropy, vol. 22, no. 3, p. 318, 2020.

[16] A. Khan, S. Ashraf, S. Abdullah, M. Qiyas, J. Luo, and S. Khan, "Pythagorean fuzzy Dombi aggregation operators and their application in decision support system," Symmetry, vol. 11, no. 3, p. 383, 2019.

[17] S. Z. Abbas, M. S. Ali Khan, S. Abdullah, H. Sun, and F. Hussain, "Cubic Pythagorean fuzzy sets and their application to multi-attribute decision making with unknown weight information," Journal of Intelligent \& Fuzzy Systems, vol. 37, no. 1, pp. 1529-1544, 2019.

[18] P. Talukdar and P. Dutta, "Distance measures for cubic Pythagorean fuzzy sets and its applications to multicriteria decision making," Granular Computing, vol. 6, no. 2, pp. 267-284, 2019.

[19] A. Fahmi, N. Yaqoob, and W. Chammam, "Maclaurin symmetric mean aggregation operators based on cubic Pythagorean linguistic fuzzy number," Journal of Ambient Intelligence and Humanized Computing, vol. 12, no. 2, pp. 1925-1942, 2020.

[20] M. B. Gorzałczany, "A method of inference in approximate reasoning based on interval-valued fuzzy sets," Fuzzy Sets and Systems, vol. 21, no. 1, pp. 1-17, 1987.

[21] Y. B. Jun, C. S. Kim, and K. O. Yang, "Annals of fuzzy mathematics and informatics," Cubic Sets, vol. 4, pp. 83-98, 2011.

[22] X. Peng and Y. Yang, "Fundamental properties of intervalvalued Pythagorean fuzzy aggregation operators," International Journal of Intelligent Systems, vol. 31, no. 5, pp. 444-487, 2016.

[23] C. Maclaurin, "A second letter toMartin Folkes, Esq.; concerning the roots of equations, with demonstration of other rules of algebra," Philosophical Transactions of the Royal Society A, vol. 36, pp. 59-96, 1729.

[24] G. Wei, C. Wei, J. Wang, H. Gao, and Y. Wei, "Some q-rung orthopair fuzzy maclaurin symmetric mean operators and their applications to potential evaluation of emerging technology commercialization," International Journal of Intelligent Systems, vol. 34, no. 1, pp. 50-81, 2019. 